\title{
Two-bidder all-pay auctions with interdependent valuations, including the highly competitive case ${ }^{1}$
}

\author{
Lucas Rentschler \\ Centro Vernon Smith de Economía Experimental \\ Universidad Francisco Marroquin \\ Guatemala, Guatemala \\ lrentschlerdufm.edu \\ Theodore L. Turocy \\ School of Economics and \\ Centre for Behavioural and Experimental Social Science \\ University of East Anglia \\ Norwich, United Kingdom \\ T.Turocy@uea.ac.uk
}

November 24, 2015

\footnotetext{
${ }^{1}$ We thank conference participants at the UECE Lisbon Meetings 2014, seminar participants at University of East Anglia, the Economic Science Institute at Chapman University, and the University of South Carolina, as well as Subhasish Modak Chowdhury, Dan Kovenock, Emiliya Lazarova, and Ron Siegel, for very useful suggestions in improving this work. The comments of the editor and two anonymous referees have been instrumental in sharpening the exposition of our results. We claim property rights over any remaining errors.
} 


\begin{abstract}
We analyze symmetric, two-bidder all-pay auctions with interdependent valuations and discrete type spaces. Relaxing previous restrictions on the distribution of types and the valuation structure, we present a construction that characterizes all symmetric equilibria. We show how the search problem this construction faces can be complex. In equilibrium, randomization can take place over disjoint intervals of bids, equilibrium supports can have a rich structure, and non-monotonicity of the equilibrium may result in a positive probability of allocative inefficiency when the value of the prize is not common. Particular attention is paid to the case in which an increase in a bidder's posterior expected value of winning the auction is likely to be accompanied by a corresponding increase for the other bidder. Such environments are "highly competitive" in the sense that the bidder's higher valuation also signals that the other bidder has an incentive to bid aggressively.
\end{abstract}

JEL Classifications: D44; D82; D72.

Keywords: contests, all-pay auctions, mixed strategies. 


\section{Introduction}

Consider an emerging industry, in which potential entrants are considering irrevocable research and development investments to develop a new product. It is believed the entrant who develops the best product will dominate. Suppose an entrant privately observes information which suggests that demand in the industry will be high, so the successful firm will obtain high profits. Higher profits are good news for the entrant - provided it is the entrant who will obtain them! If a competitor is likely to observe similar information, then the entrant may expect to face fierce competition in the research and development stage; this is bad news. Because such information is both good and bad news, determining what equilibrium behavior will look like is a potentially difficult problem.

Much existing research in all-pay auction models has ruled out this case by restricting the distributions of types such that being a "higher" type is unambiguously good news. This occurs, for example, if types are statistically independent; see e.g., Amann and Leininger (1996). In a setting with affiliated values and continuous strategy and type spaces Krishna and Morgan (1997) formulate a condition such that increasing a bidder's type is unambiguously good news for that bidder; in their words, this rules out the case that types and values are "too affiliated." With that assumption, bidding strategies and equilibrium payoffs are monotonically increasing in type. More recently, Siegel (2014) analyzes a setting with finite sets of types under a discrete analogue of the assumption of Krishna and Morgan, but without requiring affiliation. He shows that equilibrium bidding strategies are monotonic in a stochastic sense, and provides a constructive algorithm for finding equilibrium. Siegel notes that "it would be valuable to extend the analysis to...non-monotonic equilibria."

This paper carries out that extension by providing a complete analysis of symmetric equilibria in symmetric, two-bidder all-pay auctions with a finite set of types. This complements the work of Krishna and Morgan and of Siegel by bringing into focus the role that monotonicity assumptions play both in the structure of equilibria, and in the complexity of constructing an equilibrium. We provide a characterization of all symmetric equilibria, and we show that there always exists a symmetric equilibrium in which all types randomize in a piecewise-uniform way over a finite number of intervals of bids.

One way in which monotonicity may fail is when types are highly correlated. For example, suppose the two entrants in the emerging industry from the first paragraph base their judgments in part on public information, and/or use similar methodologies. Then, their information about the profitability of the market (their types) will be strongly correlated, as will their posterior assessments of the value of winning. In this sense, the contest can be thought of as being highly competitive. In addition to being economically plausible, there are models in the literature on winner-pay auctions with exactly this property. A prominent example is found in Kagel and Levin 
(1986) and Casari et al. (2007), who employ a model in which there is a common value, and conditional on that common value each bidder receives a signal about the value which is an independent draw from a uniform distribution over a small interval centered on the true value. This environment does not satisfy the Krishna-Morgan-Siegel monotonicity condition. Athey (2001) notes that the single crossing property fails in all-pay auctions with this information structure.

Relatively accurate information is not required for resulting equilibria to be non-monotonic. To illustrate this, we analyze in Section 4.3 a common-value setting in which the Krishna-MorganSiegel monotonicity requirement is not satisfied even when the private information of different types is very inaccurate.

There are therefore multiple classes of models of potential economic interest in which equilibria are not monotonic. In any case in which values are not purely common, this has important implications for the allocative efficiency of the all-pay mechanism, because it implies that the highest-value contestant need not be the one who wins the auction. It is then interesting to ask how inefficiency depends on the parameters of the information and valuation structures. Also, in settings which do not satisfy the Krishna-Morgan-Siegel monotonicity condition, equilibrium need not be unique, and equilibrium payoffs may differ for some or all types in different equilibria.

To allow us to analyze these issues, we provide an algorithm which constructs all symmetric piecewise-uniform equilibria. This algorithm can be viewed as an extension of the construction in Siegel (2014). In some settings, it is possible to have equilibria in which randomization is not piecewise-uniform over all intervals. It is also possible, under particular conditions, to have equilibria in which the equilibrium support for a type consists of an arbitrarily large number of disjoint components. We show that for any such non-piecewise-uniform or non-finite equilibrium, there exists a corresponding finite, piecewise-uniform equilibrium with similar properties. Our construction diagnoses the existence of these other types of equilibria.

The algorithm identifies which subsets of types can simultaneously include the same bid in their supports within an equilibrium behavior strategy; we call these admissible active sets. We relate possible equilibrium structures to a partial ordering over these admissible active sets; any equilibrium corresponds to some path through the graph induced by this partial ordering. When the Krishna-Morgan-Siegel monotonicity condition does not hold, this graph can become complex, and therefore the number of paths the construction must consider can grow rapidly. The graph of the partial ordering therefore helps to visualize the complexity of identifying an equilibrium in the general case.

This paper contributes to the growing literature on all-pay auctions. In a complete information environment, Baye et al. (1996) characterizes the set of equilibria. Siegel (2009) and Siegel (2010) recently greatly increased the understanding of complete information environments by allowing for a variety of asymmetries among bidders. In addition, Tullock contests, in which the highest 
bidder does not win with certainty, are the subject of a similarly growing literature; see e.g., Tullock (1980) and Cornes and Hartley (2005).

Introducing incomplete information into both all-pay auctions and Tullock contests substantially complicates analysis. The literature regarding incomplete information predominantly focuses on all-pay auctions. ${ }^{1}$ Much of the analysis considers the case of independent types. Morath and Münster (2008) demonstrate that all-pay auctions generate lower expected revenue if the vector of valuations is common knowledge relative to the case in which each bidder's valuation is private information. Amann and Leininger (1996) examine the two-bidder case under independent private values in which valuations need not be drawn from the same distribution. Kirkegaard (2007) considers comparative statics in the case of ex-ante heterogeneous bidders and finds that total effort expenditure may increase when one bidder gets weaker. Kirkegaard (2010) also considers such ex-ante asymmetry and also allows for the presence of handicapped bidders, as well as advantaged bidders whose bids are costlessly increased by an additive bonus. In all the environments considered, equilibrium is monotonic.

When types are not independent, in general a monotonicity assumption is imposed on the distribution of types which guarantees that equilibria will be monotonic. In addition to the alreadymentioned analyses of Krishna and Morgan (1997) and Siegel (2014), Harstad (1995) and Lizzeri and Persico (2000) both adopt this approach. An important exception is Lu and Parreiras (2014), who extend Amann and Leininger (1996) by allowing for correlated types and interdependent valuations in an environment with continuous type and strategy spaces. They provide a necessary and sufficient condition for the existence of monotonic equilibria. They also compute a non-monotonic equilibrium in a setting with quadratic valuations. Another exception is Einy et al. (2013), which examines common-value environments with a finite set of states of the world which determine the value of winning the auction. Bidders are ex-ante asymmetrically informed in that they differ according to their ability to distinguish between possible values of winning the auction. They characterize the equilibrium in mixed strategies and show that the probability of winning the auction and the expected bids are the same for better and less informed bidders.

The rest of the paper is organized as follows. Section 2 presents the model. Section 3 proves existence of an equilibrium, and presents the constructive algorithm. ${ }^{2}$ Section 4 analyzes some selected cases which illustrate what equilibria can look like, and illustrates that the challenge of finding equilibria can increase rapidly outside the set of environments satisfying the KrishnaMorgan-Siegel monotonicity condition. Section 5 concludes with a discussion. For conciseness in

\footnotetext{
${ }^{1}$ There are some notable exceptions. For example, the case of common-value Tullock contests with asymmetric information has been studied in Wärneryd (2003) and Wärneryd (2008).

${ }^{2} \mathrm{An}$ implementation of the construction, including analysis of all of the examples presented in this paper, is written in Python and available under the terms of the GNU General Public License from the corresponding author's website at http://www.gambit-project.org/turocy.
} 
the exposition, we relegate proofs to appendices.

\section{Model}

We consider symmetric, two-bidder all-pay auctions with a single, indivisible prize. Each bidder's type is private information, and is from a finite set $T$, with typical element $t_{k}$. After observing their types, bidders simultaneously submit bids, which can be any nonnegative real number. Each bid is irrevocably sunk, but only the bidder with the higher of the bids wins the prize. In the event of a tie the winner is determined by a fair randomization, although in symmetric equilibrium ties are a zero-probability event.

A bidder's valuation of the prize may depend on both her and her opponent's types in any arbitrary way. Let $V_{k, l}>0$ denote the posterior expected value of the prize to a bidder, conditional on being type $t_{k}$ while the other bidder is type $t_{l}$. Given that a bidder is type $t_{k}$, write the conditional probability that the other bidder is type $t_{l}$ as $h_{l \mid k}$. We assume that $h_{k \mid k}>0$ for all types $t_{k}$.

The values $V$ and conditional probabilities $h$ are sufficient to define the payoff functions for the model. In several examples, it will be convenient, for the purpose of exposition, to motivate or calculate $h$ and $V$ in terms of an underlying state of the world. When this is employed, $\Omega$ will denote the set of states of the world, with typical elements written using $\omega$ with subscripts.

We consider symmetric Bayes-Nash equilibria. A behavior strategy $\Pi$ assigns to each type $t_{k}$ a corresponding probability distribution $\Pi_{k}$ over bids. For any Lebesgue-measurable subset $B$ of bids, $\Pi_{k}: B \rightarrow \mathbb{R}_{+}$denotes the probability that the bidder will choose a bid in $B$. We endow the set of behavior strategies with the topology of weak convergence.

The expected payoff to a bidder who bids $b$, conditional on being type $t_{k}$, assuming the opponent bids according to $\Pi$, is

$$
u_{k}(b \mid \Pi)=\sum_{l: t_{l} \in T} h_{l \mid k} V_{k, l}\left[\Pi_{l}([0, b))+\frac{\Pi_{l}(\{b\})}{2}\right]-b .
$$

For notational compactness, we define the quantity $\psi_{l \mid k} \equiv h_{l \mid k} V_{k, l}$.

Siegel (2014) provides a procedure for computing equilibria in two-bidder all-pay auctions (symmetric or asymmetric), under the assumption that for every $t_{l} \in T, \psi_{l \mid k}$ is strictly increasing in $t_{k}$. This assumption is a discrete analogue of the sufficient condition for existence of symmetric and monotonic equilibrium in the case of continuous types with affiliated values identified in Krishna and Morgan (1997). We will refer to this condition as KMS. In games in which KMS is satisfied, the unique equilibrium involves piecewise-uniform randomization of bids, such that the support of the equilibrium mixed strategy is a connected interval for all types. This equilibrium is stochastically monotonic. That is, for any two types $t_{k}$ and $t_{l}$ with $k>l$, if $b$ is in the support 
of $\Pi_{l}$ and $b^{\prime}$ is in the support of $\Pi_{k}$, then $b^{\prime} \geq b$. Unlike Krishna and Morgan (1997), the results and construction by Siegel (2014), and those in this paper, does not require affiliation of types and values.

One way in which KMS may fail to hold is due to extreme correlation, either positive or negative, between private values. Consider a private-values environment with two types, in which $V_{1,1}=V_{1,0}=V_{H}$ and $V_{0,1}=V_{0,0}=V_{L}$, with $V_{H}>V_{L}$. For any $\sigma \in[0,1]$, let $h_{1 \mid 1}=h_{0 \mid 0}=\sigma$ and $h_{0 \mid 1}=h_{1 \mid 0}=1-\sigma$. The case of $\sigma=\frac{1}{2}$ corresponds to independently-determined values, with $\sigma>\frac{1}{2}$ resulting in positive correlation and $\sigma<\frac{1}{2}$ negative correlation. KMS requires that $\frac{v_{L}}{v_{H}}<\frac{\sigma}{1-\sigma}<\frac{v_{H}}{v_{L}}$, that is, the values cannot be too highly correlated, either positively or negatively. Our construction provides the equilibrium for all values of $\sigma{ }^{3}$ However, KMS is not a condition that only rules out extreme correlation. In Example 5 below, we present a model of interdependent valuations, in which bidders have identity-dependent preferences regarding the value of winning the prize. Although the bidders' values are statistically uncorrelated, nevertheless the environment does not satisfy KMS.

\section{Characterizing and constructing equilibria}

Proposition 1. In any symmetric equilibrium $\Pi$, for all types $t_{k}$, both $\Pi_{k}([0, b))$ and $u_{k}(b \mid \Pi)$ are Lipschitz-continuous functions of $b$.

Proposition 2. There exists a symmetric equilibrium.

Proof. See Appendix A for proofs of both Propositions 1 and 2

Neither KMS nor affiliation is required for existence. ${ }^{4}$ However, when KMS is not satisfied, the structure of equilibria can become quite complex. In what follows, we develop an algorithm which constructs all the symmetric equilibria in which the cumulative distribution of bids is piecewise linear for all types, and characterizes all other symmetric equilibria, if they exist. This algorithm can be viewed as an extension of the construction presented by Siegel (2014).

Given an equilibrium $\Pi$, let $\theta_{k}(\Pi)$ be the expected payoff of a bidder of type $t_{k}$. Because $u_{k}(b \mid \Pi)$ is continuous in $b$, the set of best responses $B R_{k}(\Pi)=\left\{b: u_{k}(b \mid \Pi)=\theta_{k}(\Pi)\right\}$ is a closed set. Let $E_{k}(\Pi)$ be the set of boundary points of $B R_{k}(\Pi)$ which are not isolated points. That is, $E_{k}(\Pi)=\left\{b: \exists \delta>0\right.$ s.t. $\forall 0<\varepsilon<\delta,(b-\varepsilon, b+\varepsilon) \cap B R_{k}(\Pi) \neq \emptyset \wedge(b-\varepsilon, b+\varepsilon) \cap\left(B \backslash B R_{k}(\Pi)\right) \neq$

\footnotetext{
${ }^{3}$ This is comparable to Example 1 in Siegel (2014). Our software implementation includes this model as an implemented example.

${ }^{4}$ The fact that equilibria are without atoms is a consequence both of having only two bidders, and of symmetry. Baye et al. (1996) exhibit equilibria with atoms in cases with asymmetries between bidders, and with more than two bidders. Siegel (2014) exhibit such equilibria in asymmetric two-bidder environments.
} 
$\emptyset\} .{ }^{5}$ Write $E(\Pi)=\cup_{k: t_{k} \in T} E_{k}(\Pi)$. Because in equilibrium no bidder would ever submit a positive bid without a positive probability of winning, the probability the other bidder makes a lower bid must be positive, and so inf $E(\Pi)=0$. To construct equilibria, we develop necessary conditions for an equilibrium which describe how many points $E(\Pi)$ can contain, which types have boundary points in their supports at each point in $E(\Pi)$, and, ultimately, the location of those boundary points in the space of bids.

Consider any two bids $e$ and $e+L$, with $L>0$, such that $(e, e+L) \cap E(\Pi)=\emptyset$. On any such $(e, e+L)$, define $A=\left\{t_{k} \in T: u_{k}(b \mid \Pi)=\theta_{k}(\Pi) \forall b \in(e, e+L)\right\}$. We refer to the set $A$ as the active set associated with such an interval, in the sense that the constraint $u_{k}(b \mid \Pi) \leq \theta_{k}(\Pi)$ required by equilibrium is binding or active for types in $A$. When referring to a given active set $A$, we say that $t_{k}$ is active if $t_{k} \in A$.

In any equilibrium $\Pi$ the net payoff change on $(e, e+L)$ for the types in $A$ must be zero. That is, the system of equations

$$
\sum_{l: t_{l} \in T} \psi_{l \mid k}\left[\Pi_{l}([0, e+L))-\Pi_{l}([0, e))\right]=L \forall k: t_{k} \in A
$$

must have a solution such that $\Pi_{k}([0, e+L)) \geq \Pi_{k}([0, e))$ for all $k: t_{k} \in A$, and $\Pi_{k}([0, e+L))=$ $\Pi_{k}([0, e))$ for all $k: t_{k} \in T \backslash A$. This condition does not depend on the absolute level of bids, and can be expressed using only the length $L$ and the probability masses $\mu_{k} \equiv \Pi_{k}([0, e+L))-$ $\Pi_{k}([0, e))$ expended by each type $t_{k}$.

Definition 3. A non-empty subset $A \subseteq T$ of types is an admissible active set if the system

$$
\sum_{l: t_{l} \in T} \psi_{l \mid k} \mu_{l}=L \forall k: t_{k} \in A
$$

has a solution with $L \geq 0, \mu_{k} \geq 0$ for all $k: t_{k} \in A$, and $\mu_{k}=0$ for all $k: t_{k} \in T \backslash A .{ }^{6}$ Let

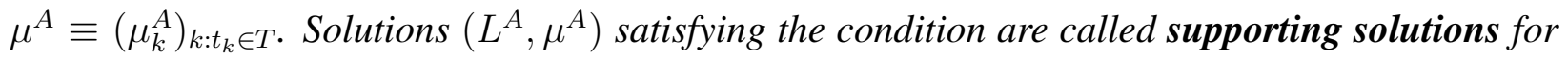
the admissible active set. Let $\mathcal{A}$ denote the set of admissible active sets.

Only sets of types which form admissible active sets can be active over a positive measure of bids in an equilibrium. Because admissibility is independent of the level of bids, determination of whether an active set is admissible can be pre-computed, ruling out possibly many active sets from consideration. ${ }^{7}$ For many games, $|\mathcal{A}| \ll 2^{|T|}$; in particular, Siegel (2014) shows that KMS implies

\footnotetext{
${ }^{5}$ Isolated points in $B R_{k}(\Pi)$ cannot be boundary points in the support of $\Pi_{k}$ because the Lipschitz-continuity of $\Pi_{k}([0, b))$ rules out mass points and singular measures. Lipschitz-continuity also ensures $E_{k}(\Pi)$ is nonempty.

${ }^{6}$ Geometrically, admissibility requires that there is an affine hyperplane containing the vectors $\{\psi \cdot \mid k\}_{t_{k} \in A}$. We thank one of the referees for suggesting this interpretation.

${ }^{7}$ Because the condition requires nonnegative solutions to a linear set of equations, Farkas' Lemma is well-suited to this task.
} 
that only singleton active sets are admissible.

Any equilibrium then necessarily satisfies

$$
\begin{aligned}
\sum_{l: t_{l} \in T} \psi_{l \mid k} \mu_{l}^{A} & =L^{A} \forall k: t_{k} \in A \forall A \in \mathcal{A} \\
\sum_{A \in \mathcal{A}} \mu_{k}^{A} & =1 \forall k: t_{k} \in T \\
\mu_{k}^{A} & \geq 0 \forall k: t_{k} \in A \forall A \in \mathcal{A} \\
\mu_{k}^{A} & =0 \forall k: t_{k} \in T \backslash A \forall A \in \mathcal{A} \\
L^{A} & \geq 0 \forall A \in \mathcal{A} .
\end{aligned}
$$

These conditions constrain, for each admissible active set $A \in \mathcal{A}$, the possible allocations of the measure of bids $L^{A}$, and the measure of probability mass $\mu_{k}^{A}$ for each type.

All admissible active sets have families of supporting solutions with at least one dimension. If the set of supporting solutions for some admissible active set $A$ has one dimension, the supporting solutions can be parameterized by the interval length $L^{A}$, and the probability mass expended by each type is proportional to $L^{A}$. It then follows that on any interval of bids on which $A$ is the active set, randomization must be uniform for all types. It is possible to have families of supporting solutions with two or more dimensions; such an example is provided in Section 4.1. In such a case randomization over an interval of bids need not be uniform. However, there always exists an equilibrium with piecewise-uniform randomization.

Proposition 4. There exists a symmetric equilibrium in which randomization is piecewise-uniform for all types.

Proof. See Appendix A.

In view of Proposition 4, in our construction we restrict attention to equilibria which involve uniform randomization on each interval. Any equilibrium with non-uniform randomization can be obtained by constructing an equilibrium with uniform randomization, and then modifying the randomization on intervals on which the active set has supporting solutions with two or more dimensions. In any equilibrium with piecewise-uniform randomization, for all types $t_{k} \in T$, the slope of the payoff function $u_{k}(b \mid \Pi)$ is well-defined and constant on each interval $(e, e+L)$ such that $(e, e+L) \cap E(\Pi)=\emptyset$.

A bid $e \in E(\Pi)$ is a point at which the active set differs for bids just above and just below $e$. Consider some point $e \in E(\Pi)$, and pick $\varepsilon>0$ small enough that $(e-\varepsilon, e+\varepsilon) \cap E(\Pi)=\{e\}$. Let $A^{+}$denote the active set on $(e, e+\varepsilon)$, and $A^{-}$denote the active set on $(e-\varepsilon, e)$. Suppose 
$t_{k} \in A^{+}$but $t_{k} \notin A^{-}$; then, $u_{k}(b \mid \Pi)$ must be increasing on $(e-\varepsilon, e) .{ }^{8}$ Similarly, suppose $t_{k} \notin A^{+}$ but $t_{k} \in A^{-}$; then $u_{k}(b \mid \Pi)$ must be decreasing on $(e, e+\varepsilon)$. These necessary conditions on an equilibrium suggest a partial ordering $\succ$ over admissible active sets.

Definition 5. Consider two admissible active sets $A$ and $A^{\prime}$. We say $A \succ A^{\prime}$ if, for all types $t_{k}$,

- If $t_{k} \in A$ and $t_{k} \notin A^{\prime}$, then there exists a supporting solution $\left(L^{A^{\prime}}, \mu^{A^{\prime}}\right)$ for $A^{\prime}$ such that $\sum_{l: t_{l} \in T} \psi_{l \mid k} \mu_{l}^{A^{\prime}}-L^{A^{\prime}}>0$.

- If $t_{k} \notin A$ and $t_{k} \in A^{\prime}$, then there exists a supporting solution $\left(L^{A}, \mu^{A}\right)$ for $A$ such that $\sum_{l: t_{l} \in T} \psi_{l \mid k} \mu_{l}^{A}-L^{A}<0 .{ }^{9}$

The partial ordering $\succ$ induces a directed graph $\Gamma$ on the admissible active sets. Any equilibrium $\Pi$ corresponds to some path through $\Gamma$. Each point $e \in E(\Pi)$ corresponds to an edge traversed in the path, with the active set at bids just above $e$ corresponding to the origin of the edge, and the active set at bids just below $e$ corresponding to the destination of the edge. In settings in which KMS is not satisfied, $\Gamma$ may become quite complex, and there will be many paths which do not correspond to an equilibrium. The exhaustive enumeration of these possible paths is where the computational complexity of identifying equilibria in the general case lies.

In a path through $\Gamma$, one or more types $t_{k}$ may be part of an active set at one node, be inactive over successive nodes, and then return to activity further down the path. If there is an equilibrium over such a path, the type will exhibit a gap in its support of bids.

Definition 6. A list $\left\{a_{j}\right\}_{j=0}^{n+1}$ of admissible active sets is a gap subpath of $\Gamma$ for type $t_{k}$ if

- $t_{k} \in a_{0}$ and $t_{k} \in a_{n+1}$

- $t_{k} \notin a_{j}$ for all $1 \leq j \leq n$;

- $a_{j-1} \succ a_{j}$ for all $1 \leq j \leq n+1$.

The set of gap subpaths for type $t_{k}$ is denoted $\mathcal{X}_{k}$, and the set of all gap subpaths is denoted $\mathcal{X}=\cup_{k: t_{k} \in T} \mathcal{X}_{k}$.

Because the graph $\Gamma$ is finite, the total number of gap subpaths is also finite.

Let $\alpha=\left\{\alpha_{j}\right\}$ denote some path through $\Gamma$. To identify whether there is an equilibrium which corresponds to $\alpha$, we extend the conditions (4) to allocate measures of bids and probability mass

\footnotetext{
${ }^{8} u_{k}(b \mid \Pi)$ cannot be decreasing; if it were, then bids in $(e-\varepsilon, e)$ would be strictly better responses for $t_{k} . u_{k}(b \mid \Pi)$ cannot be constant, because then by definition $t_{k} \in A^{-}$.

${ }^{9}$ Following the geometric interpretation, $A \succ A^{\prime}$ if, whenever $t_{k} \in A$ and $t_{k} \notin A^{\prime}$, the vector $\psi_{\cdot \mid k}$ lies above some hyperplane of $A^{\prime}$; and whenever $t_{k} \notin A$ and $t_{k} \in A$, the vector $\psi_{\cdot \mid k}$ lies below some hyperplane of $A$.
} 
of the behavior strategies to the entries in $\alpha$.

$$
\begin{aligned}
\sum_{l: t_{l} \in T} \psi_{l \mid k} \mu_{l}^{\alpha_{j}} & =L^{\alpha_{j}} \forall k: t_{k} \in \alpha_{j} \forall \alpha_{j} \in \alpha \\
\sum_{j: \alpha_{j} \in \alpha} \mu_{k}^{\alpha_{j}} & =1 \forall k: t_{k} \in T \\
\mu_{k}^{\alpha_{j}} & \geq 0 \forall k: t_{k} \in \alpha_{j} \forall \alpha_{j} \in \alpha \\
\mu_{k}^{\alpha_{j}} & =0 \forall k: t_{k} \in T \backslash \alpha_{j} \forall \alpha_{j} \in \alpha \\
L^{\alpha_{j}} & \geq 0 \forall \alpha_{j} \in \alpha .
\end{aligned}
$$

Further, it must be that there is zero net payoff change over any gap in the support of a type's strategy. Letting $\Xi_{k}=\left\{(j, n): n>j \wedge\left\{\alpha_{j^{\prime}}\right\}_{j^{\prime}=j}^{j^{\prime}=n} \in \mathcal{X}_{k}\right\}$ be the set of subpaths within $\alpha$ which correspond to a gap for type $t_{k}$, we require

$$
\sum_{j^{\prime}=j+1}^{n-1} \sum_{l: t_{l} \in T} \psi_{l \mid k} \mu_{l}^{\alpha_{j^{\prime}}}=\sum_{j^{\prime}=j+1}^{n-1} L^{\alpha_{j^{\prime}}} \forall(j, n) \in \Xi_{k} \forall t_{k} \in T .
$$

Therefore, the feasibility problem of whether a particular candidate path $\alpha$ is consistent with an equilibrium reduces to a system of linear equations and inequalities. Further, note that the solutions for each admissible active set can be pre-computed, as the set of solutions is independent of where an active set may occur in a path through $\Gamma$.

If $\Gamma$ is acyclic, all paths $\alpha$ through $\Gamma$ are finite, and the system (5) and (6) consists of a finite number of equations and inequalities in a finite number of unknowns. However, whenever there are at least three types, it is possible to construct games in which $\Gamma$ has cycles. We explore this possibility in Example 5 in subsection 4.5. When this occurs, there may be infinitely many equilibria, each one corresponding to traversing a cycle in $\Gamma$ a different number of times. However, when there is an equilibrium in which a cycle is traversed more than once, there is always a corresponding one in which each gap subpath is traversed no more than once.

Proposition 7. There exists a symmetric equilibrium, in which each type $t_{k}$ randomizes piecewiseuniformly over no more than $\left|\mathcal{X}_{k}\right|+1$ disjoint intervals of bids.

Proof. See Appendix A.

Proposition 7 then provides the final piece to ensure that the constructive algorithm terminates. If $\Gamma$ is acyclic, then all paths are finite, and the enumeration algorithm will terminate in finite time. If $\Gamma$ contains cycles, then it is enough to consider paths such that each gap subpath appears at most one time; if in traversing the graph a gap subpath is encountered a second time, then that path be discarded. In such a case, any equilibrium found involving that gap subpath will correspond to 
a family of equilibria, corresponding to paths through $\Gamma$ which visit that gap subpath any number of times.

The equilibrium search algorithm, then, consists of these steps:

1. Determine which active sets are admissible by solving (3).

2. For each admissible active set, use the solutions to (3) and inspect the slopes of the payoff functions for types not in the admissible active set to construct the graph $\Gamma$.

3. Enumerate the paths through $\Gamma$. For each path,

(a) Construct the system in (5) and (6).

(b) Determine if the system has any solutions.

(c) For each solution on some path through $\Gamma$, construct the corresponding behavior strategy by traversing the path in reverse order, setting the lower bound of the interval of bids associated with the last active set equal to zero.

(d) For each of these candidate equilibria, determine, for each type, whether the expected payoff is nonnegative, by computing the expected payoff of the highest bid submitted by the type.

\section{Examples}

\subsection{Example 1: Common values with highly correlated information}

We begin with a common-value setting. Suppose two competitors are vying to develop the technology and infrastructure to carry out mining activities on asteroids. At least initially, resource extraction in space will require high capital investments, and so there may be only room for one viable firm in the industry. While there is uncertainty about the amounts of various minerals, or useful volatiles such as water, available in asteroids, there is much available data from remote sensing and exploration missions produced by publicly-funded space exploration bodies, as well as scientific researchers. Although they will have access to very similar information, the two competitors would likely weight various sources of information in different ways, leading to estimates

for the potential economic value which would not necessarily be identical, but likely would be very similar to each other.

In the winner-pay auction literature, a model capturing the principal features of this setting has been analyzed, particularly in the study of the winner's curse, by Kagel and Levin (1986) and Casari et al. (2007). The common value of the good is drawn from a uniform distribution. 
Each bidder's type is the common value plus a conditionally independent noise term, drawn from a uniform distribution with a mean of zero. This environment does not satisfy KMS, for any size of the support of the conditional type distribution. ${ }^{10}$ Athey (2001) noted that this information structure does not satisfy the single crossing property. Wang (1991) analyzed this model with a discrete type space for the first-price auction, and showed that in the symmetric equilibrium types mix on continuous and non-overlapping intervals. In contrast, in the all-pay auction, most types randomize over multiple intervals of bids, and multiple types are are in the active set for many of these intervals.

Example 1. There are $K \geq 3$ possible states of the world, $\Omega=\left\{\omega_{1}, \ldots, \omega_{K}\right\}$, with $\omega_{k}=$ $\frac{k}{K}$. These correspond to the common value of the prize. There are $K+1$ possible types, $T=$ $\left\{t_{0}, t_{1}, \ldots, t_{K}\right\}$. Conditional on state $\omega_{k}$ being realized, each bidder is independently either type $t_{k-1}$ or $t_{k}$, with equal probability. We will refer to types $\left\{t_{1}, \ldots, t_{K-1}\right\}$ as interior types. For an interior type $t_{k}$, the conditional probability structure satisfies $h_{k-1 \mid k}=h_{k+1 \mid k}=\frac{1}{4}$ and $h_{k \mid k}=\frac{1}{2}$. For the end cases, we have $h_{1 \mid 0}=h_{0 \mid 0}=h_{K-1 \mid K}=h_{K \mid K}=h_{k \mid k}=\frac{1}{2}$. All other conditional probabilities are zero.

Turning to conditional expected values, it is useful to define $\Delta=\frac{1}{2 K}$. For any pair of interior types $t_{k-1}$ and $t_{k}$, we have $V_{k, k}=\frac{k+\frac{1}{2}}{K}=(2 k+1) \Delta$ and $V_{k-1, k}=\frac{k}{K}=2 k \Delta$. Changing either type from $t_{k-1}$ to $t_{k}$ increases the posterior expected value by $\Delta$. Types $t_{0}$ and $t_{K}$ are special because each can infer the value with certainty, and so $V_{0,0}=V_{0,1}=\frac{1}{K}=2 \Delta$ and $V_{K-1, K}=V_{K, K}=1=2 K \Delta$.

As an illustration of the operation of the constructive procedure, we first present in some detail the calculation of the equilibria of this game for the case of $K=3$. There are 15 possible active sets; of these, 12 are admissible. The graph $\Gamma$ induced by $\succ$ over these admissible active sets is shown in Figure 1a. ${ }^{11}$ Table 1 lists the admissible active sets and the corresponding supporting solutions. It also tabulates the slopes of the payoff function for types not in the active set; the signs of these determine the edges in the graph of Figure 1a. Of note is the case when all types are active. Here, the required admissibility conditions for any three types imply the fourth. Therefore, the set of supporting solutions for this active set is two-dimensional.

In this game, there is one family of equilibria, corresponding to one path through this graph, which is summarized in Table 2. The equilibrium conditions can be expressed in terms of five variables: four interval lengths $L^{\alpha_{0}}, L^{\alpha_{1}}, L^{\alpha_{2}}$, and $L^{\alpha_{3}}$, and the mass $\mu_{0}^{\alpha_{3}}$ expended on $\alpha_{3}$ by type

\footnotetext{
${ }^{10}$ Having the tails of the distribution fall to zero outside the interval makes for convenience in analysis. For KMS to be violated, it is enough to have the tails of the distribution tending to zero at a fast enough rate.

${ }^{11}$ Where we show the graphs, for compactness, we only show paths on which all types are active in at least one node, and which originate at the active set corresponding to the highest-bidding active set in the equilibrium. The node labels refer to the indices of the active types.
} 


\begin{tabular}{|c|c|c|c|c|c|c|c|c|}
\hline \multirow[b]{2}{*}{$A$} & \multicolumn{4}{|c|}{$\mu_{k}^{A}$} & \multicolumn{4}{|c|}{$\sum_{l: t_{l} \in T} \psi_{l \mid k} \mu_{l}^{A} / L^{A}-1$} \\
\hline & $t_{0}$ & $t_{1}$ & $t_{2}$ & $t_{3}$ & $t_{0}$ & $t_{1}$ & $t_{2}$ & $t_{3}$ \\
\hline$t_{3}$ & 0 & 0 & 0 & $2 L^{A}$ & -1 & -1 & $-\frac{1}{2}$ & \\
\hline$t_{2}$ & 0 & 0 & $\frac{12}{5} L^{A}$ & 0 & -1 & $-\frac{3}{5}$ & & $+\frac{1}{5}$ \\
\hline$t_{1}$ & 0 & $4 L^{A}$ & 0 & 0 & $-\frac{1}{3}$ & & $-\frac{1}{3}$ & -1 \\
\hline$t_{1} t_{3}$ & 0 & $4 L^{A}$ & 0 & $2 L^{A}$ & $-\frac{1}{3}$ & & $+\frac{0}{6}$ & \\
\hline$t_{1} t_{2}$ & 0 & $\frac{36}{11} L^{A}$ & $\frac{12}{11} L^{A}$ & 0 & $-\frac{5}{11}$ & & & $-\frac{5}{11}$ \\
\hline$t_{0}$ & $6 L^{A}$ & 0 & 0 & 0 & & $-\frac{1}{2}$ & -1 & -1 \\
\hline$t_{0} \quad t_{3}$ & $6 L^{A}$ & 0 & 0 & $2 L^{A}$ & & $-\frac{1}{2}$ & $-\frac{1}{2}$ & \\
\hline$t_{0} t_{2}$ & $6 L^{A}$ & 0 & $\frac{12}{5} L^{A}$ & 0 & & $-\frac{1}{10}$ & & $+\frac{1}{5}$ \\
\hline$t_{0} t_{1}$ & $3 L^{A}$ & $3 L^{A}$ & 0 & 0 & & & $-\frac{1}{2}$ & -1 \\
\hline$t_{0} t_{1} t_{3}$ & $3 L^{A}$ & $3 L^{A}$ & 0 & $2 L^{A}$ & & & 0 & \\
\hline$t_{0} t_{1} t_{2}$ & $5 L^{A}$ & $1 L^{A}$ & $2 L^{A}$ & 0 & & & & 0 \\
\hline$t_{0} t_{1} t_{2} t_{3}$ & $p L^{A}$ & $6 L^{A}-p$ & $p-3 L^{A}$ & $5 L^{A}-p$ & & & & \\
\hline
\end{tabular}

Table 1: Summary of admissible active sets in the common-values with highly correlated types model with $K=3$. The first group of columns summarizes the supporting solutions for each admissible active set; there is a two-dimensional family of solutions for the active set $\left\{t_{0}, t_{1}, t_{2}, t_{3}\right\}$, parameterized by $p \in[3,5]$. The second group of columns reports the slopes of the payoff functions for types not in the active set.

\begin{tabular}{|c|c|c|c|c|c|c|c|c|c|}
\hline & \multirow[b]{2}{*}{$A$} & \multicolumn{4}{|c|}{$\mu_{k}^{A}$} & \multicolumn{4}{|c|}{$\sum_{l: t_{l} \in T} \psi_{l \mid k} \mu_{l}^{A} / L^{A}-1$} \\
\hline & & $t_{0}$ & $t_{1}$ & $t_{2}$ & $t_{3}$ & $t_{0}$ & $t_{1}$ & $t_{2}$ & $t_{3}$ \\
\hline$\alpha_{0}$ & $t_{3}$ & 0 & 0 & 0 & $2 L^{\alpha_{0}}$ & -1 & -1 & $-\frac{1}{2}$ & \\
\hline$\alpha_{1}$ & $t_{2}$ & 0 & 0 & $\frac{12}{5} L^{\alpha_{1}}$ & 0 & -1 & $-\frac{3}{5}$ & & $+\frac{1}{5}$ \\
\hline$\alpha_{2}$ & $t_{1} t_{2}$ & 0 & $\frac{36}{11} L^{\alpha_{2}}$ & $\frac{12}{11} L^{\alpha_{2}}$ & 0 & $-\frac{5}{11}$ & & & $-\frac{5}{11}$ \\
\hline$\alpha_{3}$ & $t_{0} t_{1} t_{2} t_{3}$ & $p L^{\alpha_{3}}$ & $6 L^{\alpha_{3}}-p$ & $p-3 L^{\alpha_{3}}$ & $5 L^{\alpha_{3}}-p$ & & & & \\
\hline
\end{tabular}

Table 2: Path of active sets corresponding to equilibrium in the common-values with highly correlated types model with $K=3$. 


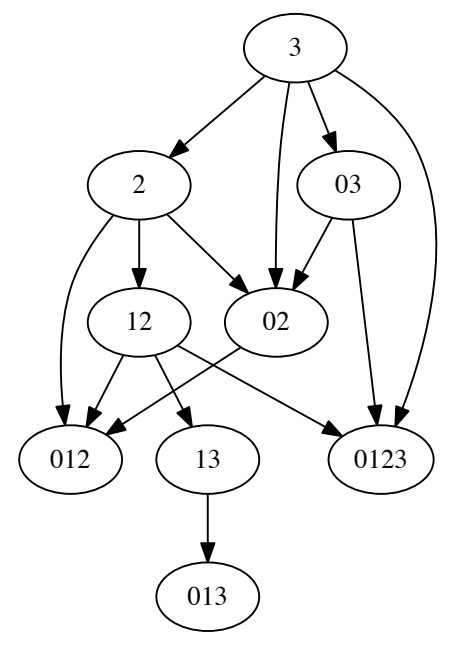

(a) The graph $\Gamma$ of admissible active sets.

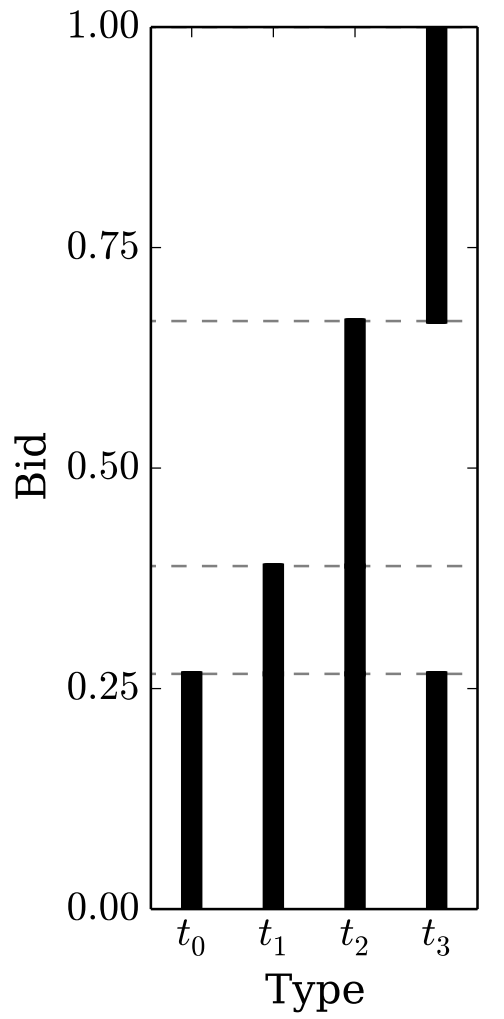

(b) Schematic of equilibrium support. Bars indicate intervals of bids on which the corresponding type is active. Horizontal dashing indicates bids at which the active set changes.

Figure 1: Visualization of admissible active sets and equilibrium in the common-values with highly correlated types model with $K=3$. 
$t_{0}$. The integrate-to-one conditions for each type are, respectively,

$$
\begin{aligned}
\mu_{0}^{\alpha_{3}} & =1 \\
\frac{36}{11} L^{\alpha_{2}}+6 L^{\alpha_{3}}-\mu_{0}^{\alpha_{3}} & =1 \\
\frac{12}{5} L^{\alpha_{1}}+\frac{12}{11} L^{\alpha_{2}}-3 L^{\alpha_{3}}+\mu_{0}^{\alpha_{3}} & =1 \\
2 L^{\alpha_{0}} & +5 L^{\alpha_{3}}-\mu_{0}^{\alpha_{3}}=1 .
\end{aligned}
$$

In addition, there is one gap subpath, for type $t_{3}$ between $\alpha_{0}$ and $\alpha_{3}$, requiring that

$$
\frac{1}{5} L^{\alpha_{1}}-\frac{5}{11} L^{\alpha_{2}}=0
$$

These equations have a unique solution, given by $\mu_{0}^{\alpha_{3}}=1, L^{\alpha_{0}}=\frac{1}{3}, L^{\alpha_{1}}=\frac{5}{18}, L^{\alpha_{2}}=\frac{11}{90}$, $L^{\alpha_{3}}=\frac{4}{15}$. Because all types are active at a bid of zero, it follows immediately that the equilibrium payoff for each type is zero. Figure $1 \mathrm{~b}$ illustrates the support of the equilibrium. For each type, the bar indicates intervals of bids on which that type is active; the type with the highest posterior estimation of the value, $t_{3}$, is active only on the highest and the lowest interval of bids, but not at intermediate bids.

The support shown in Figure $1 \mathrm{~b}$ is unique, but how the probability mass allocated to $\alpha_{3}$ is distributed is underdetermined. The most straightforward solution is uniform randomization, with densities $\left(\frac{15}{4}, \frac{9}{4}, \frac{3}{4}, \frac{5}{4}\right)$ for each of the types, respectively, over the interval $\left[0, \frac{4}{15}\right]$. Another possibility is randomization with densities $(3,3,0,2)$ on $\left[0, \frac{2}{15}\right]$, and then with densities $\left(\frac{9}{2}, \frac{3}{2}, \frac{3}{2}, \frac{1}{2}\right)$ on $\left[\frac{2}{15}, \frac{4}{15}\right]$. Indeed, any density function $\pi_{0}(b):\left[0, \frac{4}{15}\right] \rightarrow[3,5]$ with $\int_{0}^{\frac{4}{15}} \pi_{0}(b) d b=1$ corresponds to an equilibrium with densities on $\left[0, \frac{4}{15}\right]$ given by $\left(\pi_{0}(b), 6-\pi_{0}(b), \pi_{0}(b)-3,5-\pi_{0}(b)\right)$.

In the equilibria of this game, the order of active sets and the interval lengths corresponding to them are uniquely determined. Further, the portion of the behavior strategy corresponding to all but $\alpha_{3}$ is also the same for all equilibria. The total probability mass assigned to $\alpha_{3}$ is the same for all equilibria, but there are infinitely many ways in which that mass can be distributed over the corresponding interval of bids.

The number of paths through $\Gamma$ to be considered grows rapidly in $K$. For $K=8$, there are 909,238 possible paths through the graph; the graph is too large to be visualized in a figure in any practical way. Figure 2 displays the unique equilibrium support for this case. Further, the main qualitative results from the $K=3$ and $K=8$ cases can be established to hold more generally. In particular, the presence of gaps in the equilibrium support for most types, the diagonal banding of the component intervals in the supports from top right to bottom left, and the non-uniqueness of 


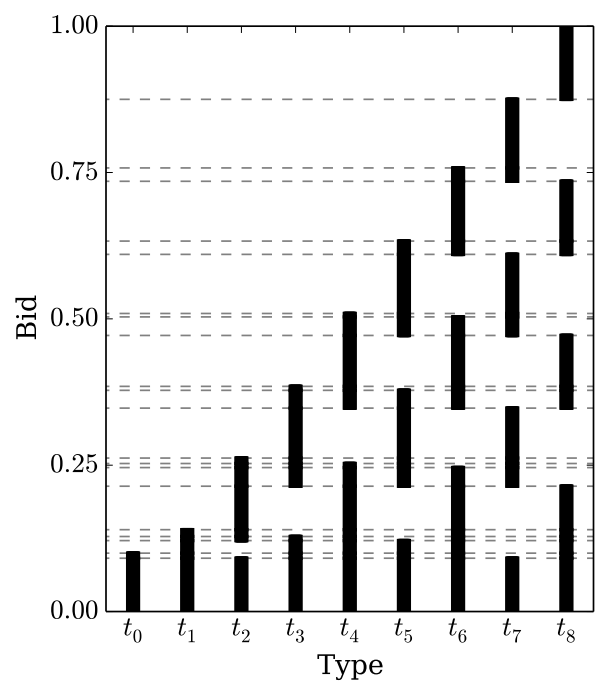

Figure 2: Schematic of equilibrium support in the common-values with highly correlated types model with $K=8$. Bars indicate intervals of bids on which the corresponding type is active. Horizontal dashing indicates bids at which the active set changes.

equilibrium behavior in the lowest interval, all remain.

We now formalize some properties of the equilibrium structure which are independent of $K$. First note that if $\mu_{k}=0$ on some interval, then the strategic calculations for types below $t_{k}$ and those above $t_{k}$ can be considered in isolation. We define an isolated group of types as a set of types $\left\{t_{k}, \ldots, t_{k^{\prime}}\right\}$ which are all active for $k^{\prime}>k$, but where $t_{k-1}$ and $t_{k^{\prime}+1}$ are inactive.

Proposition 8. In Example 1, the isolated groups in any admissible active set fall into these categories: (i) singleton types; (ii) even-parity groups of adjacent interior types; (iii) all types from $t_{0}$ to $t_{k}$ for any $0 \leq k \leq K$.

Proof. See Appendix B.

The fact that odd-parity isolated groups of interior types cannot be part of an admissible active set implies that, for any $K$ and in any equilibrium, there will be gap subpaths for some types in the corresponding path through $\Gamma$. If in moving from $\alpha_{j}$ to $\alpha_{j+1}$ type $t_{k}$ goes from active to inactive, then it must be that both $t_{k-1}$ and $t_{k+1}$ are active in $\alpha_{j+1}$.

Proposition 9. In Example 1, if an interior type $t_{k} \notin A$ for some $A \in \mathcal{A}$ and $\sum_{l: t_{l} \in T} \psi_{l \mid k} \mu_{l}^{A}-L^{A}>$ 0 on $A$, then $t_{k-1} \in A$ and $t_{k+1} \in A$.

Proof. See Appendix B. 
Finally, the set of all types is always an admissible active set. There is always a two-dimensional set of supporting solutions on this active set, but as the number of types increases, randomization on an interval corresponding to this active set necessarily tends toward uniform randomization.

Proposition 10. In Example 1, the active set consisting of all types is always admissible, and has a two-dimensional set of supporting solutions. For all $K \geq 3$, let $G^{K}$ be the game with $K$ types, and for each $K$ let $\left(L^{K}, \mu^{K}\right)$ be a supporting solution for the active set consisting of all types. Then, $\lim _{K \rightarrow \infty} \frac{\mu_{0}^{K}}{2 K L^{K}}=\ln 2$.

Proof. See Appendix B.

To close out the example, we note again that in the first-price auction with common values and the same information structure, the equilibrium is monotonic, and therefore a bidder with a higher type always wins when bidding against an opponent with a lower type. In contrast, in the all-pay auction, the lower-type bidder wins a substantial proportion of the time in such a contingency. In this model, there is a $50 \%$ chance bidders will be different types. Conditional on this event occurring, the lower-type bidder wins $12.78 \%$ of the time when $K=3$, and $17.52 \%$ of the time when $K=8$.

\subsection{Example 2: Correlated private values and multiplicity of equilibrium supports}

To affirm that the main features of the equilibrium in Example 1 are not dependent on the assumption of common values, we consider an example with similar structure, but with affiliated private values, similar to Kagel et al. (1987).

Example 2. Retain as before that there are $K \geq 3$ states of nature and $K+1$ types, and that conditional on state $\omega_{k}$, each bidder is independently either type $t_{k-1}$ or $t_{k}$ with equal probability. However, instead of the state determining the common value, now let the bidders have private values, determined by their types, with $V_{k, l}=\frac{k+1}{K+1}$.

For brevity we do not replicate the analogues of the characterization of equilibrium properties provided in Example 1. We do note, however, that the set of all types can never be an admissible active set. Consequently, some types must receive positive expected payoffs in equilibrium. In such equilibria, odd-indexed types have positive equilibrium payoffs, while even-indexed types receive zero in equilibrium. Further, each admissible active set is supported by a unique profile of densities, and therefore each equilibrium support corresponds to exactly one equilibrium behavior strategy, with piecewise-uniform randomization for every type. 

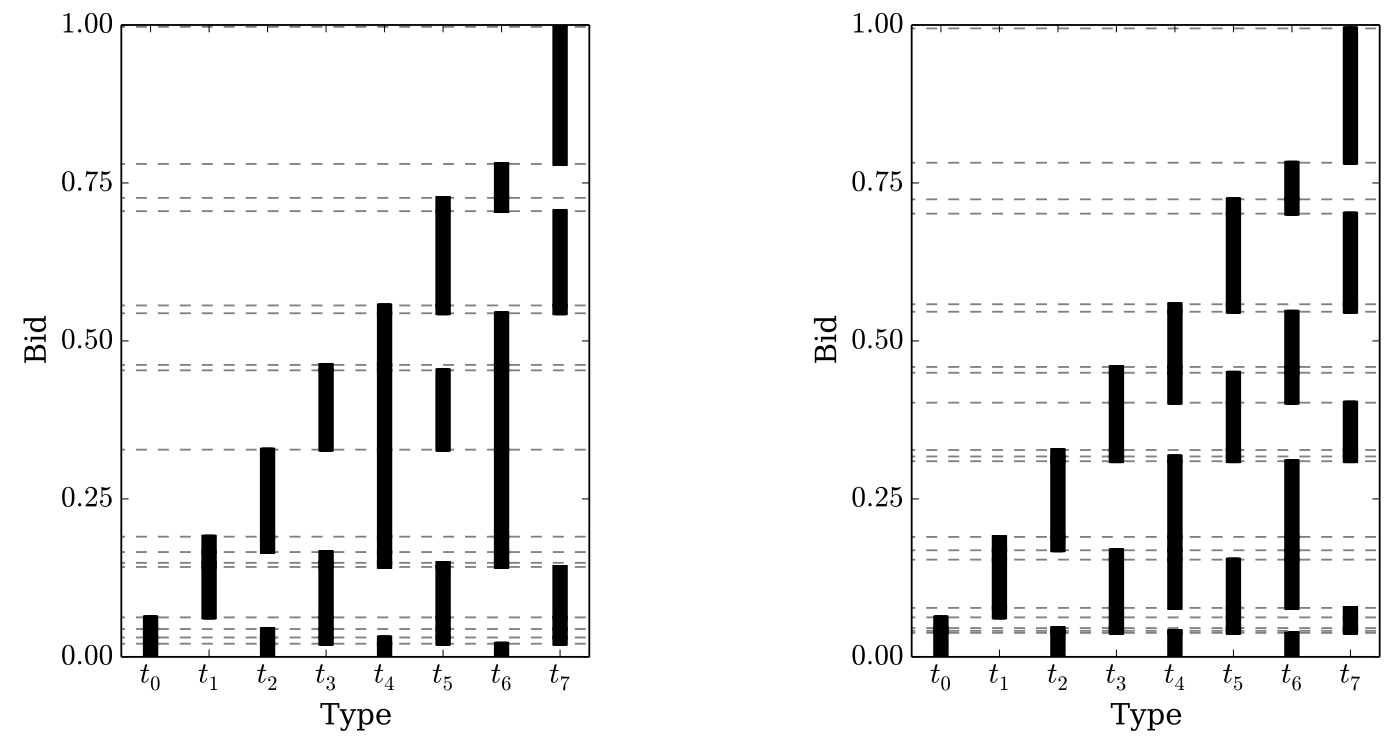

Figure 3: Schematics of the two equilibrium supports in the correlated private-values model when $K=8$. Bars indicate intervals of bids on which the corresponding type is active. Horizontal dashing indicates bids at which the active set changes.

In games in this family, there can be multiple equilibrium supports. Figure 3 depicts the two equilibrium supports for the case of $K=7$, which can be compared with Figure 2 from Example 1. The non-monotonicity of the corresponding equilibrium bidding functions implies the outcome of the auction is inefficient with positive probability, in contrast to the winner-pay auction case. As in Example 1, there is a $50 \%$ chance bidders will be different types. Conditional on this event occurring, the lower-type bidder wins $15.50 \%$ of the time in the equilibrium illustrated in the left panel of Figure 3, and $15.12 \%$ of the time in the one illustrated in the right panel. Each instance of mis-allocation has an impact of $\frac{1}{K+1}$. If the higher-value bidder always won the auction, the expected surplus would be 0.59375 . The ex-ante equilibrium loss of surplus in the equilibrium illustrated in the left panel of Figure 3 is 0.00969 and in the right panel is 0.00945, implying that $98.37 \%$ and $98.41 \%$ of the potential surplus to the bidders from winning the auction is achieved in the respective equilibria. For comparison, random allocation would lead to an efficiency of $94.74 \% .^{12}$

The assumption that each state of the world can generate only two types, and therefore that the conditional probabilities $h_{l \mid k}$ are zero for $l \notin\{k-1, k, k+1\}$, is useful for analytical tractability. This can be relaxed without changing the qualitative features of the supports shown in Figures 2

\footnotetext{
${ }^{12}$ We focus for this example on allocative efficiency. There may be other criteria for evaluating the equilibrium of an all-pay auction, for example depending on whether high bids are desired by the auction designer or society (for example, efforts in a sporting contest), or something which may be socially wasteful (for example, expenditures in lobbying games).
} 
and $3 .^{13}$

\subsection{Example 3: KMS and equilibrium selection}

Baye et al. (1996) showed that when two bidders have a commonly-known and identical value $v$ for the prize, in equilibrium the bidders randomize uniformly on the interval $[0, v]$. In this model, $v$ can either be thought of as a value which is known with certainty by both bidders, or that bidders have access to the same prior information and form the same expected value based on that information. In this example we explore the relationship between these two cases, by considering a highlycompetitive case where the values are known with almost-certainty, compared to a case where only very noisy information is received by the bidders.

Example 3a. Suppose there are $K \geq 2$ possible realizations of a common value $\left\{v_{0}, \ldots, v_{K-1}\right\}$. There are $K$ types $\left\{t_{0}, \ldots, t_{K-1}\right\}$. When the true state is $v_{k}$, a bidder is type $t_{k}$ with probability $p_{c}$. All other types are equally likely, and occur with probability $p_{w}$. We assume $p_{c}>p_{w}$ so that type $t_{k}$ can be thought of as being the "correct" type given $v_{k}$, and by necessity $p_{c}+(K-1) p_{w}=1$.

Straightforward calculations show that for all types $t_{k}$

$$
\psi_{k \mid k}=h_{k \mid k} V_{k, k}=v_{k} p_{c}^{2}+\left[\sum_{m: t_{m} \in T \backslash\left\{t_{k}\right\}} v_{m}\right] p_{w}^{2},
$$

and for all pairs of types $t_{k}$ and $t_{l}$ with $t_{k} \neq t_{l}$,

$$
\psi_{l \mid k}=h_{l \mid k} V_{k, l}=\left(v_{k}+v_{l}\right) p_{c} p_{w}+\left[\sum_{m: t_{m} \in T \backslash\left\{t_{k}, t_{l}\right\}} v_{m}\right] p_{w}^{2} .
$$

The KMS monotonicity assumption holds if and only if $\frac{p_{w}}{p_{c}}>\frac{v_{k}}{v_{k+1}}$ for all $k<K$; that is, when information about the value is relatively noisy.

Proposition 11. In Example $3 a$, there is a unique equilibrium, in which (i) each type randomizes over a connected support; (ii) on each interval, all types between $t_{l}$ and $t_{k}$ are active, for some $0 \leq l \leq k \leq K-1$.

Proof. See Appendix B.

Equilibrium in this setting has an interesting, and perhaps counterintuitive, implication about behavior when $p_{c}$ is low. Consider the case where $K=5$. Figure 4 depicts the cases of accurate

\footnotetext{
${ }^{13}$ The companion software includes an implemented example where there can be more than two types per state of the world.
} 

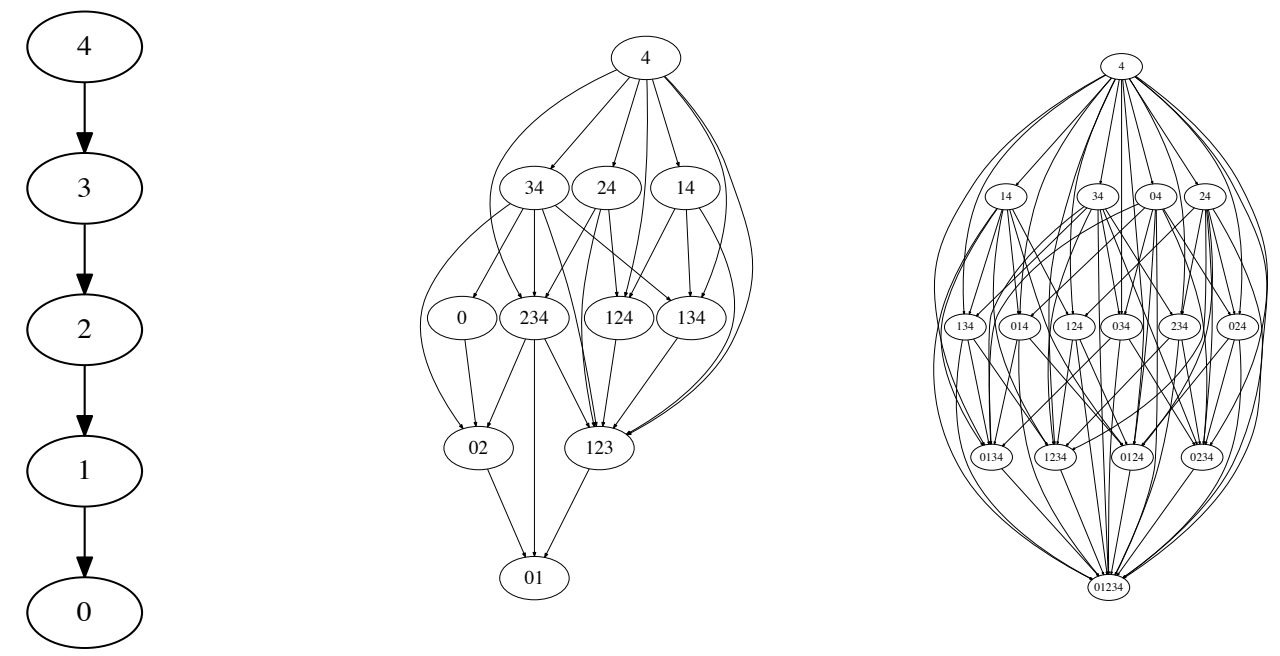

(a) Graph of admissible active sets, for $p_{c}=0.21, p_{c}=0.45$, and $p_{c}=0.99$.
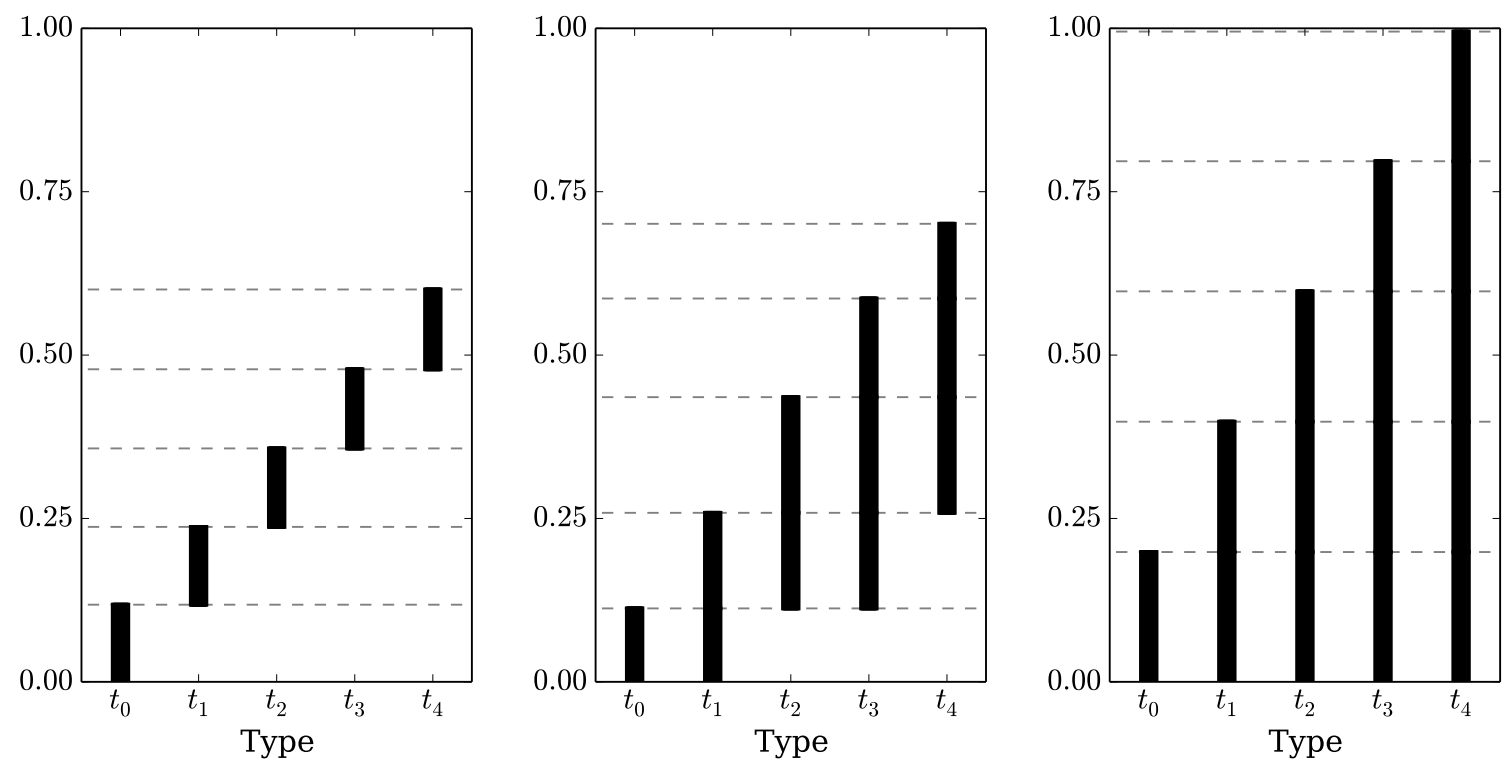

(b) Graph of equilibrium supports, for $p_{c}=0.21, p_{c}=0.45$, and $p_{c}=0.99$. Bars indicate intervals of bids on which the corresponding type is active. Horizontal dashing indicates bids at which the active set changes.

Figure 4: Complexity of the graph of admissible active sets and structure of the equilibrium of Example 3 a for the case of $K=5$, as a function of $p_{c}$. 
and inaccurate information. For each setting, the graph of admissible active sets is shown, as well as the support of the unique equilibrium.

When the probability that the type corresponds with the value is high, i.e., $p_{w} \rightarrow 0$, then there is a unique equilibrium, which converges to the Baye et al. (1996) equilibrium. For $p_{w}$ sufficiently small, a bid of zero is in the support of all types. Expected payoffs are therefore zero for all types, and so the expected sum of bids equals the expected value of the prize; it follows that the expected sum of bids is maximized by having perfectly accurate information.

However, when $p_{w} \rightarrow p_{c}$, the equilibrium does not converge to the Baye et al. (1996) equilibrium. Instead, the limiting equilibrium is separating, in that bidders who are a "higher" type always outbid those who are a "lower" one. This limiting separating equilibrium is, in fact, an equilibrium of the game with $p_{w}=p_{c}$. Although a bidder's type provides no information regarding the value of the prize, it can serve as a coordination device. All that is required for equilibrium in this case is for a bidder to believe that he is facing a uniform distribution of bids; because the type of the other bidder is not payoff-relevant, how that distribution of bids is realized as a function of the other bidder's type is not important. Therefore, any bidding strategy such that the ex-ante distribution of bids by a bidder is uniform is an equilibrium of this game.

This selection is counterintuitive in that, in the limiting case in which one's type provides no information about the value of the prize, the simplest behavior would be to use a type-independent strategy. However, for this game, and indeed for any similar common-value game where KMS is satisfied when types contain little information about the common value, the limiting equilibrium will be one in which bidders play a monotonic, fully-separating strategy. There are, however, perturbations of the Baye et al. (1996) model, in which types are arbitrarily close to being completely uninformative about the value, but nevertheless do not satisfy KMS and therefore select in the limit the type-independent equilibrium.

Example 3b. Modify Example 3a by assuming that, conditional on value $v_{k}$, with probability $1-\varepsilon$ both bidders are type $t_{k}$, for some fixed $\varepsilon>0$. Otherwise, the type is uniformly distributed over the possible types, with the randomization being realized independently for each bidder.

In the case where both bidders are the same type $t_{k}$,

$$
\psi_{k \mid k}=h_{k \mid k} V_{k, k}=(1-\varepsilon) v_{k}+\frac{\varepsilon}{K} \bar{V}
$$

If bidders are different types $t_{k} \neq t_{l}$, the case of uninformative types must have occurred, and so immediately $\psi_{k \mid l}=h_{k \mid l} V_{l, k}=\frac{\varepsilon}{K} \bar{V}$. With this structure, KMS does not hold for any value of $\varepsilon$, because for any triple of types $t_{k-1}, t_{k}$, and $t_{k+1}, \psi_{k+1 \mid k}=\psi_{k-1 \mid k}$, with $\psi_{k \mid k}$ differing from both. As a result, even when the probability of the "correct" type is only slightly greater than an 
incorrect one, but the incidence of the correct type is correlated between bidders, the equilibrium corresponding to Baye et al. (1996) is selected.

Proposition 12. In Example 3b, the following are true of admissible active sets:

1. Any subset of types forms an admissible active set;

2. For each active set, there is a unique vector of densities that is consistent with equilibrium;

3. For each active set, the payoff derivative of inactive types is always negative.

There is a unique equilibrium, in which each type $t_{k}$ randomizes over a connected interval $\left[0, B_{k}\right]$, with $B_{k}$ strictly increasing in $k$.

Proof. See Appendix B.

\subsection{Example 4: Equilibrium with non-monotonic maximum bids}

KMS requires that the good news from being a "higher" type outweighs any information that being the higher type brings about the anticipated strength of competition. In the examples considered so far, while stochastic monotonicity of the equilibrium bidding strategies fails and therefore higher types no longer win with probability one, nevertheless there is a monotonic relationship between the maximum bid in the support of each type's equilibrium bidding strategy, and that type's posterior (expected) value of the prize conditional on being that type. To bring into focus the role of the expected strength of competition, we now present an example in which there are pairs of types $t_{k}$ and $t_{l}$, such that the value of the prize is higher for $t_{l}$ than for $t_{k}$, but nevertheless the maximum equilibrium bid of $t_{l}$ is less than $t_{k}$.

Let there be $2 K$ types $\left\{t_{0}, \ldots, t_{2 K-1}\right\}$, with private values $V_{k, l}=\frac{k+1}{2 K}$ for $0 \leq k, l \leq 2 K-1$. All types are ex ante equally likely for each bidder, but types are correlated in that they tend to occur in pairs, as follows. For all $0 \leq j<K$, let $h_{2 j \mid 2 j}=h_{2 j+1 \mid 2 j+1}=p$ and $h_{2 j \mid 2 j+1}=h_{2 j+1 \mid 2 j}=p$, with $0 \leq p \leq \frac{1}{2}$. For any other pairs of types $t_{k}$ and $t_{l}, h_{l \mid k}=\frac{1-2 p}{2 K-2}$. In this setting, types $t_{0}$ and $t_{1}$ face the same distribution of types of the opposing bidder, as do types $t_{2}$ and $t_{3}$. However, the distribution of opposing types differs between $t_{1}$ and $t_{2}$. If $p$ is close to one-half, then type $t_{1}$ believes the other bidder is most likely to be type $t_{0}$ or $t_{1}$, and therefore it is most likely she has either the same or a slightly higher value than her opponent; but, type $t_{2}$ believes the other bidder is most likely type $t_{2}$ or $t_{3}$, and therefore it is most likely she has either the same or a slightly lower value than her opponent.

Figure 5 shows the unique equilibrium for the case where there are $2 K=8$ types and $p=\frac{49}{100}$. Within each pair of types, the higher type always outbids the lower type. Across pairs, type $t_{3}$ 's maximum bid is strictly greater than that of type $t_{4}$, and type $t_{5}$ 's is strictly greater than that of type $t_{6}$. 


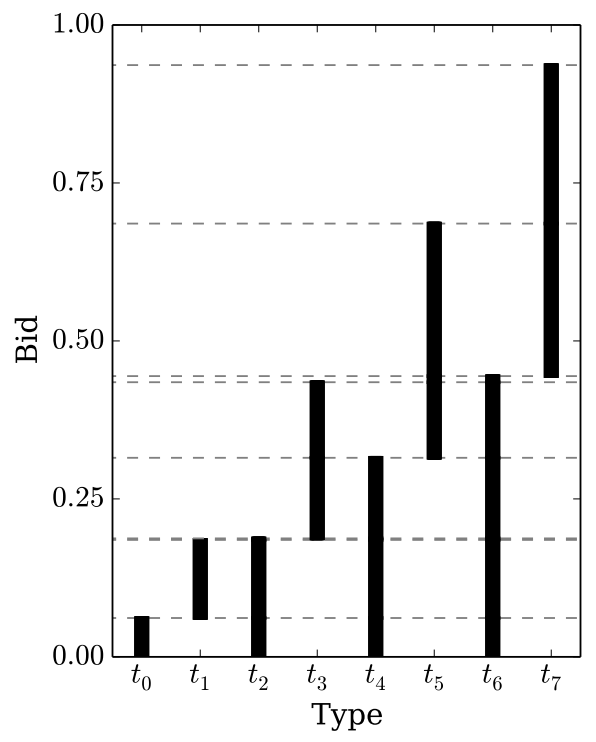

Figure 5: The support of the unique equilibrium in a private-values setting with $2 K=8$ types, where increasing from an odd-indexed to an even-indexed type implies a larger upward shift in the expected type of the other bidder. Bars indicate intervals of bids on which the corresponding type is active. Horizontal dashing indicates bids at which the active set changes.

\subsection{Example 5: Identity-dependent valuations and cycles in the active set graph}

Consider an all-pay auction among association football fans from three nations: Argentina, Brazil, and England. Suppose the satisfaction a bidder feels at winning will depend on the nation his opponent comes from. One might imagine that an Englishman would obtain the most satisfaction by winning against a Brazilian, because of Brazil's long success in international football at a game invented by the English. A Brazilian, on the other hand, might most enjoy a victory over an Argentine, due to the geographical rivalry between the teams. And, finally, an Argentine might get the most satisfaction out of a win against an Englishman, motivated by the ongoing dispute over the Falklands/Malvinas. ${ }^{14}$

Example 5. A valuation structure that encodes such rivalrous motives with three types $\left\{t_{0}, t_{1}, t_{2}\right\}$ is $V_{0,0}=V_{1,1}=V_{2,2}=2 ; V_{0,2}=V_{1,0}=V_{2,1}=3$; and $V_{0,1}=V_{1,2}=V_{2,0}=1$. Assuming for simplicity that types are independently determined and equally likely, then $\psi_{2 \mid 0}>\psi_{0 \mid 0}>\psi_{1 \mid 0}$, $\psi_{0 \mid 1}>\psi_{1 \mid 1}>\psi_{2 \mid 1}$, and $\psi_{1 \mid 2}>\psi_{2 \mid 2}>\psi_{0 \mid 2}$, and as a result there is a cycle $\left\{t_{0}\right\} \succ\left\{t_{1}\right\} \succ\left\{t_{2}\right\} \succ$

\footnotetext{
${ }^{14}$ Identity-dependent payoffs have been studied in the all-pay auction in the complete information case by Klose and Kovenock (2015).
} 
$\left\{t_{0}\right\}$ in the graph $\Gamma{ }^{15} \Gamma$ has two disconnected components: the cycle among the singleton types, and an isolated node $\left\{t_{0}, t_{1}, t_{2}\right\}$ with all types active.

This game has two families of equilibria. There is one equilibrium in which all three types randomize uniformly on the interval $[0,2]$. Then, there are infinitely many isolated equilibria which correspond to the cycle among the three singleton types. For each type $t_{k}$, the supporting solutions for the active set $A_{k} \equiv\left\{t_{k}\right\}$ require that $\frac{2}{3} \mu_{k}^{A_{k}}=L^{A_{k}}$. An equilibrium can be found using the path $\left\{A_{0}, A_{1}, A_{2}\right\}$, where $A_{2}$ is the active set on bids in $\left[0, \frac{2}{3}\right] ; A_{1}$ on $\left[\frac{2}{3}, \frac{4}{3}\right]$, and $A_{0}$ on $\left[\frac{4}{3}, 2\right]$, with the active type on each interval randomizing with density $\frac{3}{2}$. Analogous equilibria can be constructed from the paths $\left\{A_{1}, A_{2}, A_{0}\right\}$ and $\left\{A_{2}, A_{0}, A_{1}\right\}$.

Now consider the path $\alpha \equiv\left\{A_{0}, A_{1}, A_{2}, A_{0}, A_{1}, A_{2}\right\}$. The supporting solutions combined with the integrate-to-one conditions require that $L^{\alpha_{0}}+L^{\alpha_{3}}=\frac{2}{3}, L^{\alpha_{1}}+L^{\alpha_{4}}=\frac{2}{3}$, and $L^{\alpha_{2}}+L^{\alpha_{5}}=\frac{2}{3}$. There are gap subpaths for each type; the gap subpath constraint (6) for type $t_{0}$ implies $L^{\alpha_{1}}=L^{\alpha_{2}}$; for type $t_{1}$, implies $L^{\alpha_{2}}=L^{\alpha_{3}}$; and for type $t_{2}$, implies $L^{\alpha_{3}}=L^{\alpha_{4}}$. This determines that $L^{\alpha_{j}}=\frac{1}{3}$ for all $j=0,1,2,3,4,5$. One can generate a path involving as many iterations of the cycle as desired, with the result that, if the path is such that each active set appears exactly $n$ times, then the equilibrium involves each element of the path being assigned an interval of bids of length $\frac{2}{3 n}$.

The cycle does not need to be iterated an integral number of times to generate an equilibrium. The path $\alpha \equiv\left\{A_{0}, A_{1}, A_{2}, A_{0}\right\}$ requires that $L^{\alpha_{0}}+L^{\alpha_{3}}=\frac{2}{3}, L^{\alpha_{1}}=\frac{2}{3}$, and $L^{\alpha_{2}}=\frac{2}{3}$. However, there are no further constraints on $L^{\alpha_{0}}$ and $L^{\alpha_{3}}$; any allocation of the total length of $\frac{2}{3}$ between those two elements of the path corresponds to an equilibrium.

In all these cases, while there are multiple equilibria corresponding to different numbers of traversals (or partial traversals) of the cycle in $\Gamma$, all involve the same total measure of bids being allocated to each of the three singleton admissible active sets. It is in this sense that one can restrict attention to paths which feature each gap subpath at most one time; any equilibrium in which a gap subpath appears multiple times will have the same lengths of bids and probability masses assigned to each active set, but will differ only in how those are mapped onto specific bids.

\section{Conclusion}

This paper examines symmetric, two-bidder all-pay auctions with interdependent values and a general distribution of types. We assume a finite set of types, and a continuous strategy space. Our contribution is to relax monotonicity assumptions on types, which then allows, inter alia, for

\footnotetext{
${ }^{15} \mathrm{~A}$ minimum of three types is required to generate a cycle. Suppose there are only two types, fix an equilibrium $\Pi$, and consider some gap in the equilibrium support of a type $t_{k}$. It must be that there are at least two intervals involved in such a gap; on one it must be that $u_{k}(b \mid \Pi)$ is decreasing, and another on which $u_{k}(b \mid \Pi)$ is increasing. If there are only two types $t_{0}$ and $t_{1}$, there are three possible active sets: $\left\{t_{0}\right\},\left\{t_{1}\right\}$, and $\left\{t_{0}, t_{1}\right\}$. In any possible cycle involving these three active sets, no type can be inactive for two consecutive intervals.
} 
cases in which an increase in a bidder's type can be good news about the value of the prize while also being bad news about the expected level of competitiveness due to a higher valuation by the other bidder. We show existence of symmetric equilibria with piecewise-uniform randomization, and provide a characterization of all symmetric equilibria. We also show how to construct all piecewise-uniform equilibria for any setting, and how information from that construction can be used to identify the existence of other classes of equilibria.

We therefore provide answers to the discussion in the conclusion of Siegel (2014) regarding the challenge of identifying equilibria when the monotonicity condition is not satisfied. For examples with valuation and type distributions drawn from existing literature, construction of the equilibrium can be computationally intensive, and equilibria can display an exquisitely rich and complex structure. We show how using only local information, in the form of admissible active sets, can help to provide some qualitative understanding of features of possible equilibria. However, in the absence of a monotonicity condition, constructing an equilibrium involves considering global information, in the form of solving a system of linear equations over a path of admissible active sets. The systems of equations corresponding to different paths do not bear any straightforward relationship to each other in general. The complexity of the graphs that the construction traverses capture the possible complexity in the equilibrium problem, and, correspondingly, the simplicity that the monotonicity condition used by Siegel (2014) brings.

The exposition in this paper covers the symmetric case, for notational simplicity and to focus on properties of non-monotonic equilibria. An extension to the asymmetric case as considered by Siegel (2014) is straightforward. With asymmetries, an active set consists of the types for both players for which a bid is a best response, and the admissibility conditions for types of bidder 1 would depend on the probability masses allocated by bidder 2 , and vice-versa. In the asymmetric case, mass points at a bid of zero are possible, as already shown by Siegel (2014), which can be accommodated by permitting a last entry in a path to have length zero but positive masses for some types.

There are opportunities to exploit parallel processing in implementing the construction. The admissibility of each active set and its supporting solutions can be computed independently of each other, and the traversal of the graph of admissible active sets can be allocated among multiple processes. Because of this separability of tasks, speedups which are close to linear in the number of available processors should be attainable. However, in the absence of environment-specific theoretical results that allow for the pruning of the graph to rule out certain types of paths, the examples in this paper illustrate that the number of paths to consider can scale exponentially in the size of the game. In such a case a parallel implementation would in practice be able to solve slightly larger games in reasonable running times.

Also as in Siegel (2014), the ability to understand properties of candidate equilibria using the 
local information in admissible active sets depends crucially on there being only two bidders. When there are more than two bidders, equilibrium densities will depend on the bid itself, and not just on which other types are active. Our exploitation of the existence of equilibria with piecewiseuniform randomization also does not carry through to the $n$-player case, as these games do not have, in general, equilibria involving piecewise-uniform randomization.

\section{A Proofs of general existence results}

\section{Proof of Proposition 1}

First, it is straightforward that any symmetric equilibrium cannot have a mass point. Consider a behavior strategy $\Pi$ where some type $t_{k}$ has $\Pi_{k}(\{b\})=m>0$ for some bid $b$. Fix $0<\varepsilon<\psi_{k \mid k} \frac{m}{2}$. Then, the incremental benefit to bidding $b+\varepsilon$ compared to $b$ is at least $\psi_{k \mid k} \frac{m}{2}$, as a bid $b$ loses a tie with probability one-half should the other bidder's type also be $t_{k}$ and the other bidder also bids $b$, whereas $b+\varepsilon$ wins for sure in those contingencies. The incremental cost of the higher bid is $\varepsilon$. Therefore, it is strictly better for $t_{k}$ to bid $b+\varepsilon$ than $b$, and therefore $\Pi$ is not an equilibrium. Because $\Pi$ does not have mass points, the payoff function $u_{k}(b \mid \Pi)$ is continuous in $b$.

A function $f$ is Lipschitz-continuous if there exists a constant $K \geq 0$ such that $\frac{\left|f\left(x_{2}\right)-f\left(x_{1}\right)\right|}{x_{2}-x_{1}} \leq$ $K$ for all $x_{1}$ and $x_{2}$. To show that in any symmetric equilibrium $u_{k}(b \mid \Pi)$ and $\Pi_{k}([0, b))$ are Lipschitz-continuous in $b$ for all $t_{k}$, we proceed by contradiction. Pick some equilibrium $\Pi$ and some type $t_{k}$ and consider the payoff function $u_{k}(b \mid \Pi)$. Suppose $u_{k}(b \mid \Pi)$ is not Lipschitzcontinuous. Pick some $K>|T| \max _{t_{k}, t_{l} \in T} \frac{\psi_{l \mid k}}{\psi_{l \mid l}}$. Then there must exist two bids $b$ and $b+\varepsilon$ for $\varepsilon>0$ such that

$$
\begin{aligned}
\frac{\left|u_{k}(b+\varepsilon \mid \Pi)-u_{k}(b \mid \Pi)\right|}{\varepsilon} & >K \\
\left|\sum_{l: t_{l} \in T} \psi_{l \mid k}\left[\Pi_{l}([0, b+\varepsilon))-\Pi_{l}([0, b))\right]-\varepsilon\right| & >K \varepsilon .
\end{aligned}
$$

Note that the case of ties can be neglected as $\Pi$ has already been shown not to have mass points. Because $\Pi([0, b))$ is nondecreasing in $b$, the term inside the absolute value can be no smaller than $-\varepsilon$; because $K>1$ by choice, the only way for inequality (7) to be satisfied is if the term inside the absolute value is positive. This implies

$$
\left|\sum_{l: t_{l} \in T} \psi_{l \mid k}\left[\Pi_{l}([0, b+\varepsilon))-\Pi_{l}([0, b))\right]\right|>K \varepsilon .
$$

The absolute value signs on the left are superfluous, as the sum is nonnegative. One of the terms 
in the sum must be least as big as the average of all the terms; therefore there exists some type $t_{l^{\prime}}$ (which may or may not be $t_{k}$ ) such that

$$
\Pi_{l^{\prime}}([0, b+\varepsilon))-\Pi_{l^{\prime}}([0, b))>\frac{K \varepsilon}{|T| \psi_{l^{\prime} \mid k}} .
$$

Consider the behavior strategy $\Pi_{l^{\prime}}$ of $t_{l^{\prime}}$. Define

$$
\begin{aligned}
& \underline{\beta}=\sup \left\{a \geq b: \Pi_{l^{\prime}}([0, a))=\Pi_{l^{\prime}}([0, b))\right\} \\
& \bar{\beta}=\inf \left\{a \leq b+\varepsilon: \Pi_{l^{\prime}}([0, a))=\Pi_{l^{\prime}}([0, b+\varepsilon)) .\right\}
\end{aligned}
$$

Because $\Pi$ has no mass points, $\underline{\beta}<\bar{\beta}$, and in order for $\Pi$ to be an equilibrium, the continuity of $u_{l^{\prime}}(b \mid \Pi)$ requires that $u_{l^{\prime}}(\underline{\beta} \mid \Pi)=u_{l^{\prime}}(\bar{\beta} \mid \Pi)$. Inequality (8) and the definitions of $\underline{\beta}$ and $\bar{\beta}$ imply

$$
\Pi_{l^{\prime}}([0, \bar{\beta}))-\Pi_{l^{\prime}}([0, \underline{\beta}))>\frac{K \varepsilon}{|T| \psi_{l^{\prime} \mid k}} .
$$

Recalling that $\psi_{l^{\prime} \mid l^{\prime}}>0$,

$$
\begin{aligned}
\psi_{l^{\prime} \mid l^{\prime}}\left[\Pi_{l^{\prime}}([0, \bar{\beta}))-\Pi_{l^{\prime}}([0, \underline{\beta}))\right] & >\frac{K \varepsilon}{|T|} \cdot \frac{\psi_{l^{\prime} \mid l^{\prime}}}{\psi_{l^{\prime} \mid k}} \\
\sum_{l: t_{l} \in T} \psi_{l \mid l^{\prime}}\left[\Pi_{l}([0, \bar{\beta}))-\Pi_{l}([0, \underline{\beta}))\right]-(\bar{\beta}-\underline{\beta}) & >\frac{K \varepsilon}{|T|} \cdot \frac{\psi_{l^{\prime} \mid l^{\prime}}}{\psi_{l^{\prime} \mid k}}-(\bar{\beta}-\underline{\beta}) \\
u_{l^{\prime}}(\bar{\beta} \mid \Pi)-u_{l^{\prime}}(\underline{\beta} \mid \Pi) & >\left(\frac{K}{|T|} \cdot \frac{\psi_{l^{\prime} \mid l^{\prime}}}{\psi_{l^{\prime} \mid k}}-1\right) \varepsilon>0,
\end{aligned}
$$

which contradicts the assumption that $\Pi$ is an equilibrium. Therefore $u_{k}(b \mid \Pi)$ is Lipschitz-continuous in $b$ for all types $t_{k}$.

To show Lipschitz-continuity of $\Pi_{k}([0, b))$ in $b$, we again argue by contradiction. For any $K>\psi_{k \mid k}^{-1}$ there exist bids $b$ and $b+\varepsilon$ with $\varepsilon>0$ such that

$$
\Pi_{k}([0, b+\varepsilon))-\Pi_{k}([0, b))>K \varepsilon
$$

where the monotonicity of $\Pi_{k}$ permits omission of the absolute value sign. It then follows that

$$
\begin{aligned}
\psi_{k \mid k}\left[\Pi_{k}([0, b+\varepsilon))-\Pi_{k}([0, b))\right] & >\psi_{k \mid k} K \varepsilon \\
\sum_{l: t_{l} \in T} \psi_{l \mid k}\left[\Pi_{l}([0, b+\varepsilon))-\Pi_{l}([0, b))\right]-\varepsilon & >\left(\psi_{k \mid k} K-1\right) \varepsilon \\
\frac{u_{k}(b+\varepsilon \mid \Pi)-u_{k}(b \mid \Pi)}{\varepsilon} & >\psi_{k \mid k} K-1,
\end{aligned}
$$


which contradicts the Lipschitz-continuity of $u_{k}(b \mid \Pi)$.

\section{Proof of Proposition 2}

Let $G$ denote the all-pay auction game. What prohibits the immediate application of the FanGlicksberg fixed-point theorem to establish existence of an equilibrium is that the payoff function is discontinuous in the case of strategies which place a mass point on some bid for some type.

We define a sequence of perturbed games $\left\{G^{j}\right\}_{j=1}^{\infty} \cdot{ }^{16}$ These are obtained by supposing that, instead of the higher bid winning with probability one, a bidder may now win with positive probability even when his bid is slightly lower than the other bidder's. Specifically, in game $G^{j}$, we add, independent of each bidder's bid, a uniform random variable on $[-1 / j,+1 / j]$. It is these perturbed bids that are used to determine which bidder wins the auction. The cost for each bidder remains their unperturbed bids.

Let $\tilde{\Pi}_{l}^{j}\left(b ; \Pi_{l}\right)$ denote the probability that bid $b$ wins, in game $G^{j}$, conditional on the other bidder being type $t_{l}$, assuming the "intended" distribution of bids by the other bidder is given by the behavior strategy $\Pi$. Then the expected payoff to a bidder of type $t_{k}$ who bids $b$ against $\Pi$ is given by

$$
u_{k}^{j}(b \mid \Pi)=\sum_{l: t_{l} \in T} \psi_{l \mid k} \tilde{\Pi}_{l}^{j}\left(b ; \Pi_{l}\right)-b .
$$

Because of the addition of the continuous random noise, this payoff function is continuous. Therefore, the Fan-Glicksberg fixed-point theorem guarantees the existence of a symmetric equilibrium $\Pi^{j}$ in this game. For each equilibrium $\Pi^{j}$ let $\theta^{j}=\left(\theta_{k}^{j}\right)_{k: t_{k} \in T}$ denote the corresponding vector of equilibrium payoffs for each type.

Passing to a subsequence if necessary, the equilibria $\left\{\Pi^{j}\right\}$ converge to some limit $\Pi^{\star} .{ }^{17}$ Further, because the vector of equilibrium payoffs is in a compact set, we can, if necessary, consider a further subsequence such that the vector of equilibrium payoffs converges to some limit $\theta^{\star}$. We will argue that $\Pi^{\star}$ is in fact an equilibrium of the original all-pay auction game $G$.

Lemma 13. For any two bids $b_{2}>b_{1}, \frac{\Pi_{k}^{\star}\left(\left[0, b_{2}\right)\right)-\Pi_{k}^{\star}\left(\left[0, b_{1}\right)\right)}{b_{2}-b_{1}} \leq \frac{2}{\psi_{k \mid k}}$.

Proof. The argument is by contradiction. Suppose for some type $t_{k}$, there exist bids $b_{2}>b_{1}$ with $\frac{\Pi_{k}^{\star}\left(\left[0, b_{2}\right)\right)-\Pi_{k}^{\star}\left(\left[0, b_{1}\right)\right)}{b_{2}-b_{1}}>\frac{2}{\psi_{k \mid k}}$. Without loss of generality, we can assume $b_{1}$ and $b_{2}$ are points of continuity of $\Pi_{k}^{\star}([0, b)) .{ }^{18}$ For each game $G^{j}$ and corresponding equilibrium $\Pi^{j}$, let $S_{k}^{j}$ be the

\footnotetext{
${ }^{16}$ This technique is also used by Govindan and Wilson (2010), who consider the case of a continuum of types.

${ }^{17}$ Recall that the set of behavior strategies is endowed with the topology of weak convergence.

${ }^{18}$ If $b_{2}$ is not a point of continuity, the inequality will continue to be satisfied by picking $b_{2}^{\prime}=b_{2}+\delta$ for sufficiently small $\delta>0$. If $b_{1}$ is not a point of continuity, the inequality will continue to be satisfied by picking $b_{1}^{\prime}=b_{1}-\delta$ for sufficiently small $\delta>0$. To ensure the case of $b_{1}=0$ is covered, by convention we take $\Pi^{\star}([0, b))=0$ for bids $b<0$.
} 
support of $\Pi_{k}^{j}$, and define $\underline{\beta}_{k}^{j}=\inf \left\{b: b \in S_{k}^{j} \cap\left[b_{1}, b_{2}\right]\right\}$. Because $u_{k}^{j}\left(b \mid \Pi^{j}\right)$ is continuous in $b$, $u_{k}^{j}\left(\underline{\beta}_{k}^{j} \mid \Pi^{j}\right)=\theta_{k}^{j}$ and therefore $\underline{\beta}_{k}^{j}$ is a best reply for $t_{k}$ against $\Pi^{j}$ in game $G^{j}$.

Because $b_{2}$ is a point of continuity of $\Pi_{k}^{\star}, \tilde{\Pi}_{k}^{j}\left(b_{2} ; \Pi_{k}^{j}\right) \rightarrow \Pi_{k}^{\star}\left(\left[0, b_{2}\right)\right)$. We claim that

$$
\lim _{j \rightarrow \infty} \tilde{\Pi}_{k}^{j}\left(\underline{\beta}_{k}^{j} ; \Pi_{k}^{j}\right) \leq \Pi_{k}^{\star}\left(\left[0, b_{1}\right)\right)+\frac{1}{2} \Pi_{k}^{\star}\left(\left[b_{1}, b_{2}\right)\right) .
$$

If $\underline{\beta}_{k}^{j} \rightarrow b_{1}$, this follows immediately as $b_{1}$ is a point of continuity of $\Pi_{k}^{\star}$. If not, then there exists some $J$ such that, for all $j>J, b_{1}+\frac{2}{J}<\underline{\beta}_{k}^{j}<b_{2}-\frac{2}{J}$. For all these $G^{j}$ and $\Pi^{j}$, bid $\underline{\beta}_{k}^{j}$ beats bid $b_{1}$ with certainty even after the perturbation; beats a bid of $\underline{\beta}_{k}^{j}$ exactly one-half of the time; and beats all bids in $\left(\underline{\beta}_{k}^{j}, \underline{\beta}_{k}^{j}+\frac{2}{J}\right]$ with probability less than one-half. Therefore for all $j>J$,

$$
\tilde{\Pi}_{k}^{j}\left(\underline{\beta}_{k}^{j} ; \Pi_{k}^{j}\right) \leq \Pi_{k}^{j}\left(\left[0, b_{1}\right)\right)+\frac{1}{2} \Pi_{k}^{j}\left(\left[\underline{\beta}_{k}^{j}, \underline{\beta}_{k}^{j}+\frac{2}{J}\right]\right) \leq \Pi_{k}^{j}\left(\left[0, b_{1}\right)\right)+\frac{1}{2} \Pi_{k}^{j}\left(\left[\underline{\beta}_{k}^{j}, b_{2}\right)\right) .
$$

Passing to the limit as $j \rightarrow \infty$ gives (9).

Taken together, we now have that

$$
\begin{aligned}
\lim _{j \rightarrow \infty} \psi_{k \mid k}\left[\tilde{\Pi}_{k}^{j}\left(b_{2} ; \Pi_{k}^{j}\right)-\tilde{\Pi}_{k}^{j}\left(\underline{\beta}_{k}^{j} ; \Pi_{k}^{j}\right)\right] & \geq \psi_{k \mid k}\left[\Pi_{k}^{\star}\left(\left[0, b_{2}\right)\right)-\Pi_{k}^{\star}\left(\left[0, b_{1}\right)\right)-\frac{1}{2} \Pi_{k}^{\star}\left(\left[b_{1}, b_{2}\right)\right)\right] \\
& =\frac{\psi_{k \mid k}}{2}\left[\Pi_{k}^{\star}\left(\left[0, b_{2}\right)\right)-\Pi_{k}^{\star}\left(\left[0, b_{1}\right)\right)\right]>b_{2}-b_{1} .
\end{aligned}
$$

The payoff difference between $b_{2}$ and $\underline{\beta}_{k}^{j}$ in profile $\Pi^{j}$ is

$$
\begin{aligned}
u_{k}^{j}\left(b_{2} \mid \Pi^{j}\right)-u_{k}^{j}\left(\underline{\beta}_{k}^{j} \mid \Pi^{j}\right) & =\sum_{l: t_{l} \in T} \psi_{l \mid k}\left[\tilde{\Pi}_{l}^{j}\left(b_{2} ; \Pi_{l}^{j}\right)-\tilde{\Pi}_{l}^{j}\left(\underline{\beta}_{k}^{j} ; \Pi_{l}^{j}\right)\right]-\left(b_{2}-\underline{\beta}_{k}^{j}\right) \\
& \geq \psi_{k \mid k}\left[\tilde{\Pi}_{k}^{j}\left(b_{2} ; \Pi_{k}^{j}\right)-\tilde{\Pi}_{k}^{j}\left(\underline{\beta}_{k}^{j} ; \Pi_{k}^{j}\right)\right]-\left(b_{2}-b_{1}\right) .
\end{aligned}
$$

Taking the limits of both sides,

$$
\lim _{j \rightarrow \infty} u_{k}^{j}\left(b_{2} \mid \Pi^{j}\right)-u_{k}^{j}\left(\underline{\beta}_{k}^{j} \mid \Pi^{j}\right) \geq \frac{\psi_{k \mid k}}{2}\left[\Pi_{k}^{\star}\left(\left[0, b_{2}\right)\right)-\Pi_{k}^{\star}\left(\left[0, b_{1}\right)\right)\right]-\left(b_{2}-b_{1}\right)>0 .
$$

This implies that there exists some $J$ such that for all $j>J, u_{k}^{j}\left(b_{2} \mid \Pi^{j}\right)>u_{k}^{j}\left(\underline{\beta}_{k}^{j} \mid \Pi^{j}\right)$. This means that $b_{2}$ is a strictly better reply than $\underline{\beta}_{k}^{j}$ for type $t_{k}$ against $\Pi^{j}$ in game $G^{j}$, which contradicts that $\Pi^{j}$ is an equilibrium of $G^{j}$.

An immediate consequence of Lemma 13 is that $\Pi_{k}^{\star}([0, b))$ is Lipschitz-continuous in $b$, and therefore all payoff functions $u_{k}\left(b \mid \Pi^{\star}\right)$ are Lipschitz-continuous in $b$. Further, the functions $u_{k}^{j}\left(b \mid \Pi^{j}\right)$ converge to $u_{k}\left(b \mid \Pi^{\star}\right)$ (taking a subsequence if necessary). We now establish the limiting $\Pi^{\star}$ is in 
fact an equilibrium of the original game.

First, suppose that there exists some type $t_{k}$ and some bid $b$ which obtains a payoff strictly higher than $\theta_{k}^{\star}$. By continuity, there exists some $J$ such that, for all games $G^{j}$ with $j>J$, that same bid $b$ must also obtain a higher payoff than $\theta_{k}^{j}$. This contradicts that $\Pi^{j}$ is an equilibrium of $G^{j}$.

Next, consider a type $t_{k}$ and a bid $b$ which is strictly suboptimal for $t_{k}$ in $\Pi^{\star}$. Because $\Pi^{\star}$ cannot have mass points, there must be an open neighborhood around $b$ such that all bids in the neighborhood are also strictly suboptimal. Because the equilibrium payoff functions converge, it follows that there exists some $J$ such that for all games $G^{j}$ with $j>J$, all bids in some neighborhood of $b$ are suboptimal. Therefore, the corresponding equilibria $\Pi^{j}$ must place zero probability mass on the interval, and therefore the limiting equilibrium $\Pi^{\star}$ must also place zero probability mass on the interval.

\section{Proof of Proposition 4}

Consider an equilibrium $\Pi$, and suppose that over some interval $(e, e+L)$ such that $(e, e+L) \cap$ $E(\Pi)=\emptyset$, the active types randomize using a distribution that is not uniform over that interval. Because $\Pi$ is an equilibrium, equations (2) are satisfied. Let $A$ be the active set on the interval, and for each active type $t_{k} \in A$, define $\pi_{k}=\frac{\Pi_{k}([0, e+L])-\Pi_{k}([0, e])}{L}$, with $\pi_{k}=0$ for inactive types. Consider a new strategy profile $\hat{\Pi}$, such that, for any measurable set $B$ of bids, $\hat{\Pi}_{k}(B)=\pi_{k} \lambda(B \cap$ $(e, e+L))+\Pi_{k}\left(B \cap\left(\mathbb{R}_{+} \backslash(e, e+L)\right)\right)$, where $\lambda(\cdot)$ is the Lebesgue measure. We claim that $\hat{\Pi}$ must also be an equilibrium.

By construction $\hat{\Pi}$ ensures constant payoff for each active $t_{k}$ on $(e, e+L)$. For any $b_{1}, b_{2} \in$ $(e, e+L)$ and any type $t_{l}, \hat{\Pi}_{l}\left(\left[0, b_{2}\right)\right)-\hat{\Pi}_{l}\left(\left[0, b_{1}\right)\right)=\left(b_{2}-b_{1}\right) \pi_{l}$. Comparing the payoff of $b_{2}$ to that of $b_{1}$,

$$
\begin{aligned}
u_{k}\left(b_{2} \mid \hat{\Pi}\right)-u_{k}\left(b_{1} \mid \hat{\Pi}\right) & =\sum_{l: t_{l} \in A} \psi_{l \mid k}\left[\hat{\Pi}_{l}\left(\left[0, b_{2}\right)\right)-\hat{\Pi}_{l}\left(\left[0, b_{1}\right)\right)\right]-\left(b_{2}-b_{1}\right) \\
& =\sum_{l: t_{l} \in A} \psi_{l \mid k}\left(b_{2}-b_{1}\right) \pi_{l}-\left(b_{2}-b_{1}\right) \\
& =\left(b_{2}-b_{1}\right)\left[\sum_{l: t_{l} \in A} \psi_{l \mid k} \frac{\Pi_{l}([0, e+L])-\Pi_{l}([0, e])}{L}-1\right]=0
\end{aligned}
$$

because $\Pi$ being an equilibrium ensures the expression in square brackets is zero. Because $\hat{\Pi}([0, e+$ $L))=\Pi([0, e+L))$, it follows that $\theta_{k}(\hat{\Pi})=\theta_{k}(\Pi)$ for all $t_{k}$ active on $(e, e+L)$, and all bids in $(e, e+L)$ are best responses for $t_{k}$ under $\hat{\Pi}$.

Now consider any type $t_{l}$ not active on $(e, e+L)$. For any bid $b \in(e, e+L)$, because $\hat{\Pi}([0, e+$ 
$L))=\Pi([0, e+L))$ and $\hat{\Pi}([0, e))=\Pi([0, e)), u_{l}(e+L \mid \hat{\Pi})=u_{l}(e+L \mid \Pi) \leq \theta_{l}(\Pi)$ and $u_{l}(e+$ $L \mid \hat{\Pi})=u_{l}(e+L \mid \Pi) \leq \theta_{l}(\Pi)$. Because randomization on $(e, e+L)$ is uniform under $\hat{\Pi}, u_{l}(b \mid \hat{\Pi})$ is linear in $b$ on $(e, e+L)$, and therefore $u_{l}(b \mid \hat{\Pi}) \leq \theta_{l}(\Pi)$ on $(e, e+L)$. On the other hand, because all of the best responses under $\Pi$ for $t_{l}$ lie outside $(e, e+L)$, and $\hat{\Pi}([0, b)]=\Pi([0, b)]$ for all $b \notin(e, e+L)$, it follows that $\theta_{l}(\hat{\Pi})=\theta_{l}(\Pi)$.

Because $\Pi$ and $\hat{\Pi}$ differ only by redistributing probability mass within $(e, e+L)$, for all bids $b \notin(e, e+L), u_{l}(b \mid \hat{\Pi})=u_{l}(b \mid \Pi)$. Therefore, there are no bids outside $(e, e+L)$ which are best responses for any type $t_{l}$ under $\hat{\Pi}$ which are not best responses for that $t_{l}$ also under $\Pi$.

Therefore $\hat{\Pi}$ is also an equilibrium. This process can be iterated over any interval where randomization is not uniform. As a result, for any equilibrium $\Pi$, there is always some equilibrium which corresponds to the same path $\alpha$ through the graph $\Gamma$ of the game, with the same solution $\left\{\left(L^{\alpha_{j}}, \mu^{\alpha_{j}}\right)\right\}_{\alpha_{j} \in \alpha}$, and the same equilibrium payoffs to all players, where randomization is uniform along each interval of bids $(e, e+L)$ not containing a point in $E(\Pi)$.

\section{Proof of Proposition 7}

Consider an equilibrium $\Pi$ in which some type $t_{k}$ randomizes over more than $\left|\mathcal{X}_{k}\right|+1$ disjoint intervals. Let $\alpha$ be the corresponding path through $\Gamma$ and $\left\{\left(L^{\alpha_{j}}, \mu^{\alpha_{j}}\right)\right\}_{\alpha_{j} \in \alpha}$ denote the lengths and probability masses for each type along $\alpha$. We argue that we can construct another equilibrium $\hat{\Pi}$ corresponding to a different (but related) path $\hat{\alpha}$, with a solution $\left\{\left(\hat{L}^{\hat{\alpha}_{j}}, \hat{\mu}^{\hat{\alpha}_{j}}\right)\right\}_{\hat{\alpha}_{j} \in \hat{\alpha}}$.

Because each gap in the support between intervals corresponds to a gap subpath in $\Gamma$, some gap subpath $\xi \in \mathcal{X}_{k}$ must appear more than once in $\alpha$. Remembering that a gap subpath is defined as a particular list of admissible active sets visited in order, there must be a cycle in $\Gamma$. Any directed graph can be partitioned into strongly connected components; within each component, any node can be reached from any other node. Further, because the component is strongly connected, it is possible to visit all the nodes in the component without repeating a cycle. ${ }^{19}$ Finally, the partition of the graph can itself be viewed as an acyclic directed graph; once a path through the graph departs a given partition, it can never return.

Define a new path $\hat{\alpha}$ by following the path $\alpha$, but omitting any traversals of a cycle after the first one. Because any given cycle of admissible active sets is visited at most once, any given gap subpath appears in $\hat{\alpha}$ at most once. Because only multiple traversals of a cycle are omitted, $\hat{\alpha}$ visits exactly the same active sets as $\alpha$.

The solution $\left\{\left(L^{\alpha_{j}}, \mu^{\alpha_{j}}\right)\right\}_{\alpha_{j} \in \alpha}$ induces an assignment (4) of measures of bids and probability mass over active sets via (5). Remember that other than the constraints placed by the gap subpath equations (6), if an active set appears two or more times in a path, the measures of bid and

\footnotetext{
${ }^{19}$ This follows because any strongly connected directed graph has an ear decomposition.
} 
probability mass can be subdivided arbitrarily among the appearances of the active set on the path. Because $\hat{\alpha}$ visits exactly the same active sets as $\alpha$, this induced assignment from the solution on $\alpha$ also satisfies the equilibrium conditions for $\hat{\alpha}$.

In $\alpha$, for any gap subpath $\xi$ appearing more than once, the equilibrium equations require an instance of an equation of the form (6) for each appearance. If $\xi$ does not appear in $\hat{\alpha}$, then there is no constraint of the form (6) to satisfy. If it does appear, then let $(j, n)$ denote the indices in $\hat{\alpha}$ where $\xi$ appears, and let $\left(j_{i}, n_{i}\right)$ denote the indices of the $i$ th instance of $\xi$ in $\alpha$. Then, for all $1 \leq j^{\prime} \leq n-j$, let $\hat{L}^{\hat{\alpha}_{j+j^{\prime}}}=\sum_{i} L^{\alpha_{j_{i}+j^{\prime}}}$ and $\hat{\mu}_{k}^{\hat{\alpha}_{j+j^{\prime}}}=\sum_{i} \mu_{k}^{\alpha_{j_{i}+j^{\prime}}}$ for all $k: t_{k} \in T$. In words, the proposed solution on $\hat{\alpha}$ coalesces all of the bid measures and probability mass allocated to the various instances of the gap subpath in $\alpha$ to the one instance of the gap subpath in $\hat{\alpha}$. Note that this does not change the total probability mass or measure of bids assigned to each admissible active set.

Therefore, it is possible to construct a solution $\left\{\left(\hat{L}^{\hat{\alpha}_{j}}, \hat{\mu}^{\hat{\alpha}_{j}}\right)\right\}_{\hat{\alpha}_{j} \in \hat{\alpha}}$ based on $\hat{\alpha}$ using an equilibrium solution $\left\{\left(L^{\alpha_{j}}, \mu^{\alpha_{j}}\right)\right\}_{\alpha_{j} \in \alpha}$ on $\alpha$, and the solution on $\hat{\alpha}$ continues to satisfy all the requirements of equilibrium. Therefore, an equilibrium exists which traverses each gap subpath in the graph at most one time.

\section{B Other Proofs}

\section{Proofs for Section 4.1}

\section{Proof of Proposition 8}

The following facts are useful in several of the arguments below. Both can be shown by straightforward induction arguments.

Fact (Fact EVEN). Let $A$ be an admissible active set, and suppose $\left\{t_{k}, \ldots, t_{k+2 j-1}\right\}$ are interior types which comprise an isolated group within $A$, for any $1 \leq k<K$ and any $j>0$. Then, supporting solutions for A satisfy

$$
\frac{k}{2}\left(\mu_{k-1}^{A}+\mu_{k}^{A}\right)=\frac{k+2 j}{2}\left(\mu_{k+2 j-1}^{A}+\mu_{k+2 j}^{A}\right) .
$$

Fact (Fact ODD). Let $A$ be an admissible active set, and suppose $\left\{t_{k}, \ldots, t_{k+2 j}\right\}$ are interior types which comprise an isolated group within $A$, for any $1 \leq k<K$ and any $j \geq 0$. Then, supporting solutions for A satisfy

$$
\frac{k}{2}\left(\mu_{k-1}^{A}+\mu_{k}^{A}\right)=\frac{L_{A}}{\Delta}-\frac{k+2 j+1}{2}\left(\mu_{k+2 j}^{A}+\mu_{k+2 j+1}^{A}\right) .
$$


Lemma 14. No isolated group of an odd number of interior types can be part of an admissible active set.

Proof. Let $A$ be an admissible active set, and suppose $\left\{t_{k}, \ldots, t_{k+2 j}\right\}$ is an isolated group of types in $A$, for any $j \geq 1$. For all $i<j$, the admissibility equation for type $t_{k+2 i}$ from (3), combined with Fact EVEN, gives

$$
k \mu_{k}^{A}+(k+2 i+1)\left(\mu_{k+2 i}^{A}+\mu_{k+2 i+1}^{A}\right)=2 \Delta^{-1} L^{A} .
$$

For all $i<j$, the admissibility equation for type $t_{k+2 i-1}$, combined with Fact ODD, gives

$$
k \mu_{k}^{A}+(k+2 i)\left(\mu_{k+2 i-1}^{A}+\mu_{k+2 i}^{A}\right)=0 .
$$

In matrix form, the system of equations required to satisfy all the associated admissibility conditions can be written $C \mu^{A}=d$, with

$$
C=\left[\begin{array}{cccccc}
2 k+1 & k+1 & 0 & 0 & \cdots & 0 \\
k & -(k+2) & -(k+2) & 0 & \cdots & 0 \\
k & 0 & k+3 & k+3 & \cdots & 0 \\
\vdots & \vdots & \ddots & \ddots & \ddots & \vdots \\
k & 0 & \cdots & 0 & -(k+2 j) & -(k+2 j) \\
k & 0 & \cdots & \cdots & 0 & k+2 j+1
\end{array}\right], d=\left[\begin{array}{c}
2 \Delta^{-1} L^{A} \\
0 \\
2 \Delta^{-1} L^{A} \\
\vdots \\
0 \\
2 \Delta^{-1} L^{A}
\end{array}\right]
$$

By Farkas' Lemma, this system has no nonnegative solution for $\mu^{A}$ if and only if there exists some vector $y$ such that $C^{T} y \geq 0$ and $d^{T} y<0$. The matrix $C^{T}$ does not have full rank, so there will be multiple solutions to $C^{T} y=0$. We can write $C^{T} y=0$ row-wise as

$$
\begin{aligned}
(2 k+1) y_{1}+k \sum_{m=2}^{2 j+1} y_{m} & =0 \\
(k+2 i-1) y_{2 i-1}-(k+2 i) y_{2 i} & =0 \quad \forall 1 \leq i \leq j \\
-(k+2 i) y_{2 i}+(k+2 i+1) y_{2 i+1} & =0 \forall 1 \leq i<j
\end{aligned}
$$

The equations generated by the last two lines imply

$$
y_{n}=\left(\frac{k+2 j+1}{k+n}\right) y_{2 j+1} \forall 2 \leq n \leq 2 j
$$

To obtain $d^{T} y<0$ we only require that the sum of the odd-indexed entries in $y$ be negative. We 
have

$$
\begin{aligned}
\sum_{i=1}^{j} y_{2 i-1} & =-\frac{k}{2 k+1} \sum_{i=1}^{j} y_{2 i}+\frac{k+1}{2 k+1} \sum_{i=1}^{j} y_{2 i+1} \\
& =-\frac{k}{2 k+1} \sum_{i=1}^{j} \frac{k+2 j+1}{k+2 i} y_{2 j+1}+\frac{k+1}{2 k+1} \sum_{i=1}^{j} \frac{k+2 j+1}{k+2 i+1} y_{2 j+1} . \\
& =\frac{k+2 j+1}{2 k+1} y_{2 j+1} \sum_{i=1}^{j}\left[-\frac{k}{k+2 i}+\frac{k+1}{k+2 i+1}\right] .
\end{aligned}
$$

As the term in square brackets is positive, the sign of $d^{T} y$ is determined by the sign of $y_{2 j+1}$. Because $y_{2 j+1}$ can be chosen freely because $C^{T} y$ does not have full rank, choosing any $y_{2 j+1}<0$ generates the required condition. Therefore, the original system does not have a solution with nonnegative $\mu^{A}$, and the claim follows.

Lemma 15. Any isolated group of all types between $t_{0}$ and $t_{m}$ can be part of an admissible active set, for any $0 \leq m \leq K$. For any such group, for all $k \leq m$, the supporting solution satisfies $\mu_{k-1}^{A}+\mu_{k}^{A}=\frac{L^{A}}{k \Delta}$.

Proof. We proceed by induction. For the base case $k=0$, given an admissible active set $A$, the admissibility equation generated by type $t_{0}$ can be written $\Delta\left(\mu_{0}^{A}+\mu_{1}^{A}\right)=L^{A}$. For the induction step, assume the claim is true for $k-1$, and consider type $t_{k}$. Taking the admissibility condition for $t_{k}$ with $k<K$ and applying the induction hypothesis,

$$
\begin{aligned}
\frac{k}{2}\left(\mu_{k-1}^{A}+\mu_{k}^{A}\right)+\frac{k+1}{2}\left(\mu_{k}^{A}+\mu_{k+1}^{A}\right) & =\frac{L^{A}}{\Delta} \\
\frac{k}{2} \times \frac{1}{k \Delta}+\frac{k+1}{2}\left(\mu_{k}^{A}+\mu_{k+1}^{A}\right) & =\frac{L^{A}}{\Delta} \\
\mu_{k}^{A}+\mu_{k+1}^{A} & =\frac{L^{A}}{(k+1) \Delta},
\end{aligned}
$$

which establishes the result for $k$ and completes the induction step. For $t_{K}$ the solution can be confirmed by direct calculation.

Now consider type $t_{m-1}$. We have that $\mu_{m-1}^{A}+\mu_{m}^{A}=\frac{L^{A}}{m \Delta}$. This, and the admissibility equation for type $t_{m}$ constitute a $2 \times 2$ linear system of equations. The unique solution of this system is $\mu_{m}^{A}=\frac{L^{A}}{\Delta(m+1)}$ and $\mu_{m-1}^{A}=\frac{L^{A}}{\Delta m}-\frac{L^{A}}{\Delta(m+1)}$. Note that both $\mu_{m-1}^{A}$ and $\mu_{m}^{A}$ are positive. Further, given that for any type $t_{k}$ with $k<m, \mu_{k}^{A}=\frac{L^{A}}{(m+1) \Delta}-\mu_{k+1}^{A}$ all the masses $\mu_{k}^{A}$ in the solution are strictly positive.

Lemma 16. No isolated set of all types from $t_{k}$ to $t_{K}$ with $0<k<K$ may be simultaneously part of an admissible active set. 
Proof. The proof is by contradiction. Let $A$ be an admissible active set. By direct calculation, $t_{K-1}$ and $t_{K}$ cannot comprise an isolated group within $A$. Now suppose that all types from $t_{k}$ to $t_{K}$ do comprise such an isolated group within $A$, with $0<k<K-1$. Consider type $t_{K-1}$. If $K-k$ is even, Fact EVEN tells us that

$$
\frac{k}{2} \mu_{k}^{A}=\frac{K}{2}\left(\mu_{K-1}^{A}+\mu_{K}^{A}\right)
$$

Because $\mu_{K-1}^{A}+\mu_{K}^{A}=2 L^{A}, \mu_{k}^{A}=\frac{L^{A}}{2 k \Delta}$. By Fact ODD, we know that

$$
\frac{k}{2} \mu_{k}^{A}=\frac{L^{A}}{\Delta}-\left(\frac{k+1}{2}\right)\left(\mu_{k}^{A}+\mu_{k+1}^{A}\right) .
$$

Solving for $\mu_{k+1}^{A}$ leaves us with $\mu_{k+1}^{A}=\frac{L^{A}(2 k-1)}{2 k \Delta(k+1)}$. By Fact EVEN we know that

$$
\frac{k}{2} \mu_{k}^{A}=\frac{k+2}{2}\left(\mu_{k+1}^{A}+\mu_{k+2}^{A}\right)
$$

Solving for $\mu_{k+2}^{A}$ leaves us with $\mu_{k+2}^{A}=\frac{L^{A}}{2(k+2) \triangle}-\frac{L^{A}(2 k-1)}{2 k \triangle(k+1)}$, which is only positive if $k<1$.

If $K-k$ is odd then Fact ODD tells us that

$$
\frac{k}{2} \mu_{k}^{A}=\frac{L^{A}}{\Delta}-\left(\frac{K}{2}\right)\left(\mu_{K-1}^{A}+\mu_{K}^{A}\right)
$$

Solving for $\mu_{k+1}^{A}$ as above yields $\mu_{k+1}^{A}=\frac{-L^{A}}{k(k+1) \Delta}<0$.

\section{Proof of Proposition 9}

Fix some type $t_{k}$. Observe that among all admissible active sets $A, \frac{\mu_{k}^{A}}{L^{A}}$ is largest when $t_{k}$ is an isolated type in $A$. So, it is enough for us to consider two cases: $t_{k-1} \notin A$ and $t_{k+1} \in A$ as an isolated type, and $t_{k+1} \notin A$ and $t_{k-1} \in A$ as an isolated type.

First suppose $t_{k-1} \notin A$ and $t_{k+1} \in A$ is an isolated type. Then the admissibility equation for $t_{k+1}$ reduces to $\mu_{k+1}^{A}=\frac{2}{2 k+3} \frac{L^{A}}{\Delta}$, and $\psi_{k+1 \mid k} \mu_{k+1}^{A}-L^{A}=\frac{L^{A}}{2} \times \frac{2 k+2}{2 k+3}-L^{A}<0$, which is a contradiction. Next suppose $t_{k+1} \notin A$ and $t_{k-1} \in A$ is an isolated type. Then the admissibility equation for $t_{k-1}$ reduces to $\mu_{k-1}^{A}=\frac{2}{2 k-1} \frac{L^{A}}{\Delta}$, and $\psi_{k-1 \mid k} \mu_{k-1}^{A}-L^{A}=\frac{k}{2 k-1} L^{A}-L^{A}<0$, which is a contradiction.

Therefore, both $t_{k-1}$ and $t_{k+1}$ must be active, as claimed. When both are active,

$$
\psi_{k-1 \mid k} \mu_{k-1}^{A}+\psi_{k+1 \mid k} \mu_{k+1}^{A}-L^{A}=\frac{L^{A}}{2} \times \frac{8 k(k-1)-2}{4 k(k-1)-3}-L^{A}
$$


which is positive for $k>1$.

\section{Proof of Proposition 10}

Consider the active set $A$ consisting of all types $\left\{t_{0}, \ldots, t_{K}\right\}$. The conditions for admissibility lead to a degeneracy; if the conditions for any $K$ of the types are satisfied, then the admissibility condition for the other type is automatically satisfied as well. In this case an earlier claim has shown that $\mu_{k-1}^{A}+\mu_{k}^{A}=\frac{L^{A}}{k \Delta}$ for all $k$.

For any given choice of $\mu_{0}^{A}$, the recursive relationship can be unrolled to show that $\mu_{k}^{A}=$ $\frac{L^{A}}{k \Delta}-\mu_{k-1}^{A}$ and $\mu_{k}^{A}=\mu_{k-2}^{A}-\frac{L^{A}}{k(k-1) \Delta}$. We can then state that

$$
\begin{aligned}
\mu_{2 k}^{A} & =\mu_{0}^{A}-\frac{L^{A}}{\Delta} \sum_{j=1}^{k} \frac{1}{2 j(2 j-1)} \\
\mu_{2 k+1}^{A} & =\mu_{1}^{A}-\frac{L^{A}}{\Delta} \sum_{j=1}^{k} \frac{1}{2 j(2 j+1)} .
\end{aligned}
$$

Remembering that $\mu_{0}^{A}+\mu_{1}^{A}=\frac{L^{A}}{\Delta}$, in order to ensure that $\mu_{2 k}^{A} \geq 0$ and $\mu_{2 k+1}^{A} \geq 0$,

$$
\sum_{j=1}^{k} \frac{1}{2 j(2 j-1)} \leq \frac{\mu_{0}^{A}}{2 K L^{A}} \leq 1-\sum_{j=1}^{k} \frac{1}{2 j(2 j+1)}
$$

As $k$ increases, expression on the left increases monotonically, and the one on the right decreases monotonically. Therefore, for given $K$, the types $t_{K-1}$ and $t_{K}$ are the ones which establish the upper and lower bounds on $\frac{\mu_{0}^{A}}{L^{A}}$, and there is a range of $\frac{\mu_{0}^{A}}{L^{A}}$ consistent with equilibrium. As $k \rightarrow \infty$, both the left and right sides of the inequality coverge to $\ln 2$. Therefore, for a finely discretized type space, randomization on this interval is "almost" uniform.

\section{Proofs for Section 4.3}

\section{Proof of Proposition 11}

Let $A$ be an admissible active set, and consider two types $t_{k}$ and $t_{l}$; here and throughout this proof assume $k>l$. Direct calculation shows that

$$
\begin{aligned}
\sum_{m: t_{m} \in T} \psi_{m \mid k} \mu_{m}^{A}-\sum_{m: t_{m} \in T} \psi_{m \mid l} \mu_{m}^{A}= & \left(v_{k} p_{c}-v_{l} p_{w}\right)\left(p_{c}-p_{w}\right) \mu_{k}^{A}+\left(v_{k} p_{w}-v_{l} p_{c}\right)\left(p_{c}-p_{w}\right) \mu_{l}^{A} \\
& +\left(v_{k}-v_{l}\right) p_{w}\left(p_{c}-p_{w}\right) \sum_{m: t_{m} \in T \backslash\left\{t_{k}, t_{l}\right\}} \mu_{m}^{A}
\end{aligned}
$$


The first and third terms on the right side are always positive. The middle term is positive if $\frac{p_{w}}{p_{c}}>\frac{v_{l}}{v_{k}}$, that is, if the probability that a bidder's type corresponds to the value with too high a probability relative to the ratio of values. If this inequality holds for $l=k-1$ and all $k$, then KMS is satisfied, and therefore only singleton types can be admissible active sets.

Consider some path $\alpha$ through the graph $\Gamma$ of admissible active sets. For each entry $\alpha_{j}$ in the path, let $\lambda_{j} \equiv \min \left\{k: t_{k} \in \alpha_{j}\right\}$ be the smallest type index active in $\alpha_{j}$. Then, $\lambda_{j}$ is nonincreasing in $j$. To see this, suppose that $t_{k}$ is active and $t_{l}$ is inactive. Because $\mu_{l}^{A}=0$, the difference in (10) is negative, and therefore $\sum_{m: t_{m} \in T} \psi_{m \mid l} \mu_{l}^{A}<L^{A}$. Therefore, between any two consecutive entries $\alpha_{j}$ and $\alpha_{j+1}$, in order for $\lambda_{j} \notin \alpha_{j+1}$, a type with a smaller index must be in $\alpha_{j+1}$.

Next, suppose both $t_{k}$ and $t_{l}$ are active. Then it must be that

$$
\left(v_{k} p_{c}-v_{l} p_{w}\right) \mu_{k}^{A}+\left(v_{k} p_{w}-v_{l} p_{c}\right) \mu_{l}^{A}<0
$$

which implies that

$$
\frac{\mu_{k}^{A}}{\mu_{l}^{A}}<\frac{v_{l} p_{c}-v_{k} p_{w}}{v_{k} p_{c}-v_{l} p_{w}}<1
$$

and therefore $\mu_{k}^{A}<\mu_{l}^{A}$.

Now consider the case where, for some $j, t_{k} \in \alpha_{j}$ and $t_{k} \notin \alpha_{j+1}$. We claim that for all active sets $\alpha_{j^{\prime}}, j^{\prime}>j$

$$
\left(v_{k} p_{w}-v_{\lambda_{j^{\prime}}} p_{c}\right)\left(p_{c}-p_{w}\right) \mu_{\lambda_{j^{\prime}}}^{\alpha_{j^{\prime}}}+\left(v_{k}-v_{\lambda_{j^{\prime}}}\right) p_{w}\left(p_{c}-p_{w}\right) \sum_{m: t_{m} \in T \backslash\left\{t_{k}, t_{\lambda_{j^{\prime}}}\right\}} \mu_{m}^{\alpha_{j^{\prime}}}>0
$$

This must be true by construction on $j^{\prime}=j+1$ because $t_{k}$ becomes inactive in moving from $\alpha_{j}$ to $\alpha_{j+1}$, and $\alpha$ is a path through $\Gamma$. Because $\lambda_{j}$ is nonincreasing, both terms in the sum must be nondecreasing in $j$. Therefore, once a type $t_{k}$ becomes inactive, it is never active in any subsequent active set along $\alpha$, and therefore the support of type $t_{k}$ 's strategy is connected.

Finally, any active set in $\alpha$ contains all types between $t_{l}$ and $t_{k}$ for some $l \leq k$. Suppose not, and $t_{l}$ and $t_{k}$ are active for some $l<k$ but some type $t_{n}$ with $l<n<k$ is not. Because types must exit in decreasing order of their indices, the number of entries in $\alpha$ on which $t_{n}$ is active must be fewer than $t_{l}$. However, for each $\alpha_{j} \in \alpha, \mu_{n}^{\alpha_{j}}<\mu_{l}^{\alpha_{j}}$. Because $\sum_{\alpha_{j} \in \alpha} \mu_{l}^{\alpha_{j}}=1$, then $\sum_{\alpha_{j} \in \alpha} \mu_{n}^{\alpha_{j}}<1$, which violates the equilibrium conditions and forms the contradiction. 


\section{Proof of Proposition 12}

Let $A$ be an admissible active set. For any type $t_{k}$,

$$
\sum_{m: t_{m} \in T} \psi_{m \mid k} \mu_{m}^{A}-L^{A}=\frac{\varepsilon \bar{V}}{K} \sum_{m: t_{m} \in T \backslash\left\{t_{k}\right\}} \mu_{m}^{A}+(1-\varepsilon) v_{k} \mu_{k}^{A}-L^{A} .
$$

Active sets consisting of a single type are always admissible, so we consider the case when $A$ is not a singleton. Fix any $t_{k} \in A$. For each $t_{l} \in A$, with $k \neq l$, the admissibility equations for $A$ imply

$$
\frac{\varepsilon \bar{V}}{K} \sum_{m: t_{m} \in T \backslash\left\{t_{k}\right\}} \mu_{m}^{A}+(1-\varepsilon) v_{k} \mu_{k}^{A}=\frac{\varepsilon \bar{V}}{K} \sum_{m: t_{m} \in T \backslash\left\{t_{l}\right\}} \mu_{m}^{A}+(1-\varepsilon) v_{l} \mu_{l}^{A},
$$

and therefore

$$
v_{k} \mu_{k}^{A}=v_{l} \mu_{l}^{A}
$$

Substituting into the admissibility equation for $t_{k}$,

$$
\frac{\varepsilon \bar{V}}{K} \sum_{m: t_{m} \in A \backslash\left\{t_{k}\right\}} \frac{v_{k}}{v_{m}} \mu_{k}^{A}+(1-\varepsilon) v_{k} \mu_{k}^{A}=L^{A} .
$$

Pick some $L^{A}>0$; there is then some $\mu_{k}^{A}>0$ such that (12) is satisfied. For all other $t_{l} \in A$, determine $\mu_{l}^{A}>0$ via (11). Therefore, there is a solution to the admissibility conditions for $A$. As we have placed no restrictions on the types in $A$, it follows that all subsets of $T$ are admissible.

Now, suppose that $t_{k} \in A$ but $t_{l} \notin A$. Then,

$$
\sum_{m: t_{m} \in T} \psi_{m \mid k} \mu_{m}^{A}-\sum_{m: t_{m} \in T} \psi_{m \mid l} \mu_{m}^{A}=v_{k} \mu_{k}^{A}-v_{l} \mu_{l}^{A}=v_{k} \mu_{k}^{A}>0
$$

Therefore, $\sum_{m: t_{m} \in T} \psi_{m \mid l} \mu_{m}^{A}<L^{A}$. The implication of this is that along any path $\alpha$ through the graph $\Gamma$ of admissible active sets, if a type $t_{k} \in \alpha_{j}$, then $t_{k} \in \alpha_{j^{\prime}}$ for all $j^{\prime} \geq j$. As a result, in any equilibrium, a type $t_{k}$ randomizes over some connected interval $\left[0, B_{k}\right]$ of bids, where $B_{k}$ is nondecreasing in $k$. To show that $B_{k}$ is strictly increasing in $k$, we proceed by contradiction. Suppose there is an equilibrium with $B_{k}=B_{l}$ for some $k>l$. Then, types $t_{k}$ and $t_{l}$ are active on the same entries in the corresponding path $\alpha$. For each $\alpha_{j} \in \alpha$, the supporting solution must satisfy $\mu_{l}^{\alpha_{j}}=\frac{v_{k}}{v_{l}} \mu_{k}^{\alpha_{j}}$, so therefore $\mu_{l}^{\alpha_{j}}>\mu_{k}^{\alpha_{j}}$. However, this contradicts the equilibrium requirement that both $\sum_{j: \alpha_{j} \in \alpha} \mu_{k}^{\alpha_{j}}=1$ and $\sum_{j: \alpha_{j} \in \alpha} \mu_{l}^{\alpha_{j}}=1$. 


\section{References}

Erwin Amann and Wolfgang Leininger. Asymmetric all-pay auctions with incomplete information: The two-player case. Games and Economic Behavior, 14(1):1-18, 1996.

Susan Athey. Single crossing properties and the existence of pure strategy equilibria in games of incomplete information. Econometrica, 69(4):861-889, 2001.

Michael R. Baye, Dan Kovenock, and Casper G. de Vries. The all-pay auction with complete information. Economic Theory, 8:291-305, 1996.

Marco Casari, John C. Ham, and John H. Kagel. Selection bias, demographic effects, and ability effects in common value auction experiments. American Economic Review, 97(4):1278-1304, 2007.

Richard Cornes and Roger Hartley. Asymmetric contests with general technologies. Economic Theory, 26(4):923-946, 2005.

Ezra Einy, Ori Haimanko, Ram Orzach, and Aner Sela. Common-value all-pay auctions with asymmetric information. CEPR Discussion Paper No. DP9315, 2013.

Srihari Govindan and Robert Wilson. Existence of equilibria in all-pay auctions. Stanford Graduate School of Business Research Paper Series, No. 2058, 2010.

Ronald M. Harstad. Privately informed seekers of an uncertain rent. Public Choice, 83(1):81-93, 1995.

John H. Kagel and Dan Levin. The winner's curse and public information in common value auctions. American Economic Review, 76(5):894-920, 1986.

John H. Kagel, Ronald M. Harstad, and Dan Levin. Information impact and allocation rules in auctions with affiliated private values: A laboratory study. Econometrica, 55(6):1275-1304, 1987.

René Kirkegaard. Comparative statics and welfare in heterogeneous contests: Bribes, caps, and performance thresholds. Working paper 702, Brock University Department of Economics, 2007.

René Kirkegaard. Favoritism in asymmetric contests: Head starts and handicaps. In Proceedings of the Behavioral and Quantitative Game Theory Conference on Future Directions, page 45. ACM, 2010. 
Bettina Klose and Dan Kovenock. The all-pay auction with complete information and identitydependent externalities. Economic Theory, 59:1-19, 2015.

Vijay Krishna and John Morgan. An analysis of the war of attrition and the all-pay auction. Journal of Economic Theory, 72:343-362, 1997.

Alessandro Lizzeri and Nicola Persico. Uniqueness and existence of equilibrium in auctions with a reserve price. Games and Economic Behavior, 30(1):83-114, 2000.

Jingfeng Lu and Sérgio O. Parreiras. Asymmetric all-pay auctions, monotone and non-monotone equilibrium. Working Paper, 2014.

Florian Morath and Johannes Münster. Private versus complete information in auctions. Economics Letters, 101(3):214-216, 2008.

Ron Siegel. All-pay contests. Econometrica, 77(1):71-92, 2009.

Ron Siegel. Asymmetric contests with conditional investments. American Economic Review, 100 (5):2230-60, 2010.

Ron Siegel. Asymmetric all-pay auctions with interdependent valuations. Journal of Economic Theory, 2014.

Gordon Tullock. Efficient rent seeking. In James M. Buchanan, Robert D. Tollison, and Gordon Tullock, editors, Toward a theory of the rent seeking society. Texas A\&M University Press, College Station, TX, 1980.

Ruqu Wang. Common-value auctions with discrete private information. Journal of Economic Theory, 54(2):429-447, 1991.

Karl Wärneryd. Information in conflicts. Journal of Economic Theory, 110(1):121-136, 2003.

Karl Wärneryd. Multi-player contests with asymmetric information. Economic Theory, 51(2): 277-287, 2008. 